\title{
ANALYSIS OF FULLY DISCRETE FEM FOR MISCIBLE DISPLACEMENT IN POROUS MEDIA WITH BEAR-SCHEIDEGGER DIFFUSION TENSOR
}

\author{
WENTAO CAI, BUYANG LI, YANPING LIN, AND WEIWEI SUN
}

\begin{abstract}
Fully discrete Galerkin finite element methods are studied for the equations of miscible displacement in porous media with the commonly-used Bear-Scheidegger diffusion-dispersion tensor:

$$
D(\mathbf{u})=\gamma d_{m} I+|\mathbf{u}|\left(\alpha_{T} I+\left(\alpha_{L}-\alpha_{T}\right) \frac{\mathbf{u} \otimes \mathbf{u}}{|\mathbf{u}|^{2}}\right) .
$$

Previous works on optimal-order $L^{\infty}\left(0, T ; L^{2}\right)$-norm error estimate required the regularity assumption $\nabla_{x} \partial_{t} D(\mathbf{u}(x, t)) \in L^{\infty}\left(0, T ; L^{\infty}(\Omega)\right)$, while the Bear-Scheidegger diffusion-dispersion tensor is only Lipschitz continuous even for a smooth velocity field $\mathbf{u}$. In terms of the maximal $L^{p}$ regularity of fully discrete finite element solutions of parabolic equations, optimal error estimate in $L^{p}\left(0, T ; L^{q}\right)$-norm and almost optimal error estimate in $L^{\infty}\left(0, T ; L^{q}\right)$-norm are established under the assumption of $D(\mathbf{u})$ being Lipschitz continuous with respect to $\mathbf{u}$.
\end{abstract}

Keywords. miscible displacement in porous media, Bear-Scheidegger diffusion-dispersion tensor, finite element method, maximal $L^{p}$-regularity, error estimate

\section{Introduction}

The incompressible flow of binary miscible fluid in porous media is governed by the miscible displacement equations

$$
\begin{aligned}
& \gamma \frac{\partial c}{\partial t}-\nabla \cdot(D(\mathbf{u}) \nabla c)+\mathbf{u} \cdot \nabla c=\hat{c} q_{I}-c q_{I}, \\
& \nabla \cdot \mathbf{u}=q_{I}-q_{P}, \quad \mathbf{u}=-\frac{k(x)}{\mu(c)} \nabla p,
\end{aligned}
$$

where $\mathbf{u}$ and $p$ are the velocity and pressure of the fluids mixture, respectively, and $c$ is the concentration of one fluid. In this model, $k(x)$ is the permeability of the porous medium, $\mu(c)$ the concentration-dependent viscosity, $\gamma$ the porosity of the medium, $q_{I} \geq 0$ and $q_{P} \geq 0$ the given injection and production sources, respectively, and $\hat{c}$ the concentration in the injection source. A popular diffusion-dispersion tensor $D(\mathbf{u})=\left[D_{i j}(\mathbf{u})\right]_{d \times d}$ used in reservoir simulations and underground oil exploration is the Bear-Scheidegger model (cf. [6, 44])

$$
D(\mathbf{u})=\gamma d_{m} I+|\mathbf{u}|\left(\alpha_{T} I+\left(\alpha_{L}-\alpha_{T}\right) \frac{\mathbf{u} \otimes \mathbf{u}}{|\mathbf{u}|^{2}}\right),
$$

where $d_{m}>0$ denotes the molecular diffusion, and $\alpha_{L}$ and $\alpha_{T}$ the constant longitudinal and transversal dispersivities of the isotropic porous medium, respectively. We consider (1.1)-(1.2) in a bounded smooth domain $\Omega \subset \mathbb{R}^{d}$, with $d \in\{2,3\}$, up to time $T$, subject to the no-flux boundary conditions

$$
\mathbf{u} \cdot \mathbf{n}=0 \quad \text { and } \quad D(\mathbf{u}) \nabla c \cdot \mathbf{n}=0 \quad \text { on } \partial \Omega \times(0, T],
$$

with the given initial condition

$$
c(x, 0)=c_{0}(x) \text { for } x \in \Omega .
$$

Numerical methods and analysis for the miscible displacement system (1.1)-(1.5) have been investigated extensively in the last several decades, and numerical simulations have been done for various engineering applications, e.g., [10, 13, 14, 47, 48, 49]. A traditional approach to establish the optimal $L^{\infty}\left(0, T ; L^{2}\right)$-norm error estimate is based on an elliptic Ritz projection $\mathbf{R}_{h}(t): H^{1}(\Omega) \rightarrow$ 
$S_{h}^{r}$ onto the finite element space, defined by (see [50])

$$
\left(D(\mathbf{u}(\cdot, t)) \nabla\left(\phi-\mathbf{R}_{h} \phi\right), \nabla \varphi_{h}\right)=0, \quad \text { for all } \phi \in H^{1}(\Omega) \text { and } \varphi_{h} \in S_{h}^{r} .
$$

Most previous works on optimal $L^{\infty}\left(0, T ; L^{2}\right)$ error estimates of Galerkin type FEMs for (1.1)-(1.5) follow this way, which requires the following estimate of the Ritz projection:

$$
\left\|\partial_{t}\left(c-\mathbf{R}_{h} c\right)\right\|_{L^{2}\left(0, T ; L^{2}\right)} \leq C h^{r+1} .
$$

The estimate above was established by Wheeler [50] under the regularity assumption

$$
\left\|\nabla_{x} \partial_{t} D(\mathbf{u}(x, t))\right\|_{L^{\infty}\left(0, T ; L^{\infty}\right)} \leq C
$$

for a general nonlinear parabolic equation. However, less attention was paid to the regularity of the Bear-Scheidegger diffusion-dispersion tensor. It was shown in [45] that $D(\mathbf{u})$ is Lipschitz continuous in $\mathbf{u}$. In a more recent work 31, a counter example was presented to show that even for a smooth velocity field it may hold

$$
\nabla_{x} \partial_{t} D(\mathbf{u}(x, t)) \notin L^{p}\left(\Omega_{T}\right) \text { for any } p \geq 1 .
$$

Clearly, the Bear-Scheidegger dispersion model may not satisfy the regularity condition (1.8) and therefore, optimal $L^{\infty}\left(0, T ; L^{2}\right)$ error estimates of fully discrete Galerkin-Galerkin FEMs, Galerkinmixed FEMs and many other numerical methods for this model have not been well investigated in this case.

In this article, we study the commonly-used Bear-Scheidegger diffusion-dispersion model by a linearized fully discrete Galerkin FEM and establish an optimal $L^{p}\left(0, T ; L^{q}\right)$ error estimate, together with an almost optimal $L^{\infty}\left(0, T ; L^{q}\right)$ error estimate. The key to our analysis is the discrete maximal $L^{p}$-regularity ( $L^{p}$-stability) of fully discrete finite element solutions of the parabolic equations

$$
\begin{cases}\partial_{t} \phi-\nabla \cdot(a \nabla \phi)+\phi=f-\nabla \cdot \mathbf{g} & \text { in } \Omega, \\ a \nabla \phi \cdot \mathbf{n}=\mathbf{g} \cdot \mathbf{n} & \text { on } \partial \Omega, \\ \phi(x, 0)=\phi_{0}(x) & \text { for } x \in \Omega .\end{cases}
$$

In the last several decades, great efforts have been devoted to the maximal $L^{p}$-stability estimates, e.g., see [8, 9, 16, 17, 19, 26, 27, 36, 37, 39, 40, 42, 43, and references therein. A straightforward

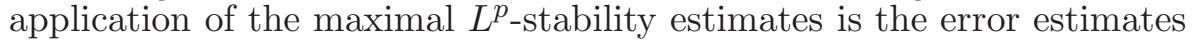

$$
\begin{aligned}
& \left\|\mathbf{P}_{h} \phi-\phi_{h}\right\|_{L^{p}\left(0, T ; L^{q}\right)} \leq C\left(\left\|\mathbf{P}_{h} \phi_{0}(x)-\phi_{h}(0)\right\|_{L^{q}}+\left\|\phi-\mathbf{R}_{h} \phi\right\|_{L^{p}\left(0, T ; L^{q}\right)}\right), \\
& \left\|\mathbf{P}_{h} \phi-\phi_{h}\right\|_{L^{\infty}\left(0, T ; L^{q}\right)} \leq C\left\|\mathbf{P}_{h} \phi_{0}(x)-\phi_{h}(0)\right\|_{L^{q}}+C \ln (2+1 / h)\left\|\mathbf{P}_{h} \phi-\phi\right\|_{L^{\infty}\left(0, T ; L^{q}\right)},
\end{aligned}
$$

with $p, q \in(1, \infty)$, where $\phi_{h}$ is the finite element solution of (1.9), $\mathbf{P}_{h}$ is the $L^{2}$-projection operator onto the finite element space $S_{h}^{r}$, and $\mathbf{R}_{h}$ the Ritz projection operator associated with the elliptic operator $\mathcal{L}=-\nabla \cdot(a \nabla)+1$. Early works on such $L^{p}\left(0, T ; L^{q}\right)$ and $L^{\infty}\left(0, T ; L^{q}\right)$ stability estimates were done mainly for spatially semi-discrete finite element solutions of linear parabolic equations with sufficiently smooth time-independent coefficients, e.g., $a_{i j}=a_{i j}(x) \in C^{2+\alpha}(\bar{\Omega})$. The extension to time-independent Lipschitz continuous coefficients $a_{i j}=a_{i j}(x) \in W^{1, \infty}(\Omega)$ was presented in [28]. Further extensions to fully discrete finite element solutions were done in [22, 23, 27] for linear autonomous parabolic equations and in 32] for linear nonautonomous parabolic equations (with coefficients $a_{i j}=a_{i j}(x, t)$ ). The former relies on the semigroup approach which is applicable only for a problem with time-independent coefficients, and the latter uses a perturbation technique together with a duality argument.

The $L^{p}\left(0, T ; L^{q}\right)$ approach has apparent advantages over the traditional $L^{\infty}\left(0, T ; L^{2}\right)$ estimate in dealing with nonlinear parabolic equations. Recently, analysis on semi-discrete nonlinear parabolic equations was presented by several authors, see [17, 31] for semi-discrete finite element methods and [2, 3, 24] for time discrete systems. However, no analysis has been done for fully discrete Galerkin FEMs for nonlinear physical equations. The $L^{p}\left(0, T ; L^{q}\right)$ analysis of a fully discrete FEM for nonlinear parabolic equations is much different from the analysis of time-discrete systems. In this paper, we apply the $L^{p}\left(0, T ; L^{q}\right)$ approach to commonly-used linearized fully discrete Galerkin finite element methods for the nonlinear miscible displacement problem (1.1)-(1.5) with the Bear-Scheidegger diffusion-dispersion tensor to establish optimal $L^{p}\left(0, T ; L^{q}\right)$ and almost 
optimal $L^{\infty}\left(0, T ; L^{q}\right)$ error estimates. More important is that our analysis illustrates a fundamental tool in establishing optimal error estimates of commonly-used fully discrete Galerkin FEMs for nonlinear physical equations with more general diffusion coefficients.

\section{Main results}

For $q \in[1, \infty]$ and any integer $k \geq 0$, we denote by $W^{k, q}=W^{k, q}(\Omega)$ the usual Sobolev spaces of functions defined on $\Omega$, with the abbreviations $L^{q}=W^{0, q}$ and $H^{k}=W^{k, 2}$; see [1]. The dual space of $W^{k, q}$ is denoted by $\widetilde{W}^{-k, q^{\prime}}$, with the notation $q^{\prime}=q /(q-1)$ and the abbreviation $\widetilde{H}^{-k}=\widetilde{W}^{-k, 2}$. For any integer $k \geq 0$ and $\alpha \in(0,1)$, we denote by $C^{k, \alpha}$ the space of functions whose partial derivatives up to $k^{\text {th }}$-order are Hölder continuous with the exponent $\alpha$.

Let $0=t_{0}<t_{1}<\cdots<t_{N}=T$ be a uniform partition of the interval $[0, T]$ for some integer $N$, with the step size $t_{n}-t_{n-1}=\tau=T / N$. For any sequence of functions $\left\{f^{n}\right\}_{n=0}^{N}$, we define

$$
\begin{aligned}
& D_{\tau} f^{n}:=\frac{f^{n}-f^{n-1}}{\tau}, \\
& \left\|f^{m}\right\|_{L^{p}(X)}:= \begin{cases}\left(\sum_{n=1}^{m} \tau\left\|f^{n}\right\|_{X}^{p}\right)^{\frac{1}{p}}, & p \in[1, \infty), \\
\max _{1 \leq n \leq m}\left\|f^{n}\right\|_{X}, & p=\infty,\end{cases}
\end{aligned}
$$

for certain Sobolev space $X$. The norm $\left\|f^{m}\right\|_{L^{p}(X)}$ is simply the $L^{p}(0, m \tau ; X)$ norm of the piecewise constant function which takes the value $f_{n}$ on each interval $\left(t_{n-1}, t_{n}\right]$.

Let $\Omega \subset \mathbb{R}^{d}$, with $d \in\{2,3\}$, be a bounded domain with smooth boundary $\partial \Omega$, and let $\mathcal{T}_{h}$ be a shape-regular and quasi-uniform triangulation of $\Omega$ into triangles or tetrahedra which fit the boundary $\partial \Omega$ exactly, with possibly curved triangles or tetrahedra near on the boundary. We denote by $h$ the mesh size of triangulation, and define the following finite element spaces:

$$
\begin{aligned}
& S_{h}^{r}=\left\{\phi_{h} \in H^{1}(\Omega): \phi_{h} \text { is a polynomial of degree } r \text { on each triangle (or tetrahedra) }\right\}, \\
& \dot{S}_{h}^{2}=\left\{\phi_{h} \in S_{h}^{2}: \int_{\Omega} \phi_{h} \mathrm{~d} x=0\right\} .
\end{aligned}
$$

We consider a linearized and stabilized fully-discrete FEM for (1.1)-(1.5), which seeks $P_{h}^{n-1} \in \stackrel{S}{h}_{h}^{2}$ and $\mathcal{C}_{h}^{n} \in S_{h}^{1}$ such that

$$
\begin{aligned}
& \left(\frac{k(x)}{\mu\left(\mathcal{C}_{h}^{n-1}\right)} \nabla P_{h}^{n-1}, \nabla v_{h}\right)=\left(q_{I}^{n-1}-q_{P}^{n-1}, v_{h}\right), \quad \forall v_{h} \in \stackrel{S}{h}_{h}^{2}, \quad n=1, \ldots, N+1, \\
& \left(\gamma D_{\tau} \mathcal{C}_{h}^{n}, w_{h}\right)+\left(D\left(\mathbf{U}_{h}^{n-1}\right) \nabla \mathcal{C}_{h}^{n}, \nabla w_{h}\right)+\left(\frac{1}{2}\left(q_{I}^{n}+q_{P}^{n}\right) \mathcal{C}_{h}^{n}, w_{h}\right) \\
& +\frac{1}{2}\left(\mathbf{U}_{h}^{n-1} \cdot \nabla \mathcal{C}_{h}^{n}, w_{h}\right)-\frac{1}{2}\left(\mathbf{U}_{h}^{n-1} \mathcal{C}_{h}^{n}, \nabla w_{h}\right)=\left(\hat{c} q_{I}^{n}, w_{h}\right), \quad \forall w_{h} \in S_{h}^{1}, \quad n=1, \ldots, N,
\end{aligned}
$$

where

$$
\mathbf{U}_{h}^{n-1}=-\frac{k(x)}{\mu\left(\mathcal{C}_{h}^{n-1}\right)} \nabla P_{h}^{n-1},
$$

and $\mathcal{C}_{h}^{0}=\Pi_{h} c(\cdot, 0)$, with $\Pi_{h}$ being the Lagrange interpolation operator onto $S_{h}^{1}$.

We assume that $q_{I}, q_{P}, \hat{c} \in C\left([0, T] ; L^{\infty}(\Omega)\right), k \in W^{2, \infty}(\Omega), \mu \in W^{2, \infty}(\mathbb{R}), k_{0} \leq k(x) \leq k_{1}$, $\mu_{0} \leq \mu(c) \leq \mu_{1}$, and the system (1.1)-(1.5) has a unique solution satisfying

$$
\|c\|_{C\left([0, T] ; W^{2, q}\right)}+\left\|\partial_{t} c\right\|_{C\left([0, T] ; W^{1, q}\right)}+\left\|\partial_{t t} c\right\|_{C\left([0, T] ; \widetilde{W}^{-1, q}\right)}+\|p\|_{C\left([0, T] ; W^{3, q}\right)} \leq K .
$$

This only guarantees the Lipschitz continuity $D(\mathbf{u}) \in L^{\infty}\left(0, T ; W^{1, \infty}\right) \cap W^{1, \infty}\left(0, T ; L^{\infty}\right)$, instead of (1.8), for the Bear-Scheidegger diffusion-dispersion tensor (1.3). Our main result is presented in the following theorem, with the notations

$$
c^{n}=c\left(\cdot, t_{n}\right), \quad p^{n}=p\left(\cdot, t_{n}\right), \quad \text { and } \quad \mathbf{u}^{n}=\mathbf{u}\left(\cdot, t_{n}\right) .
$$


Theorem 2.1. Suppose that the system (1.1)-(1.5) has a unique solution $(c, \mathbf{u}, p)$ satisfying (2.4) for some $q \in(d, \infty)$. Then the finite element system (2.1)-(2.3) admits a unique solution $\left(P_{h}^{n}, \mathcal{C}_{h}^{n}\right)$, $n=1, \ldots, N$, satisfying

$$
\left\|P_{h}^{n}-p^{n}\right\|_{L^{p}\left(W^{1, q}\right)}+\left\|\mathbf{U}_{h}^{n}-\mathbf{u}^{n}\right\|_{L^{p}\left(L^{q}\right)}+\left\|\mathcal{C}_{h}^{n}-c^{n}\right\|_{L^{p}\left(L^{q}\right)} \leq C_{p, q}\left(\tau+h^{2}\right),
$$

for any $p \in(1, \infty)$, where $C_{p, q}$ is a constant, independent of $n, \tau$ and $h$ and dependent upon $p, q$.

Corollary 2.2. Under the assumptions of Theorem 2.1, it holds that

$$
\left\|P_{h}^{n}-p^{n}\right\|_{L^{\infty}\left(W^{1, q}\right)}+\left\|\mathbf{U}_{h}^{n}-\mathbf{u}^{n}\right\|_{L^{\infty}\left(L^{q}\right)}+\left\|\mathcal{C}_{h}^{n}-c^{n}\right\|_{L^{\infty}\left(L^{q}\right)} \leq C_{\epsilon}\left(\tau^{1-\epsilon}+h^{2-\epsilon}\right),
$$

for an arbitrary small $\epsilon>0$.

The rest of this paper is devoted to the proofs of Theorem 2.1 and Corollary 2.2. The main difficulty iis to prove an upper bound for $\left\|\mathcal{C}_{h}^{n}\right\|_{W^{1, \infty}}$ in order to control the nonlinear terms involved in the analysis. To this end, we adopt the the error splitting approach developed in [29, 30] and the discrete maximal $L^{p}$-regularity of parabolic equations developed in [22, 23, 28, 31, 32]. By this approach, we first prove in Section 4 that the semi-discretization in time has sufficient regularity uniformly with respect to the time-step size, i.e.,

$$
\left\|D_{\tau} \mathcal{C}^{N}\right\|_{L^{p}\left(L^{q}\right)}+\left\|\mathcal{C}^{N}\right\|_{L^{p}\left(W^{2, q}\right)} \leq C_{p, q}
$$

where $C_{p, q}$ is a constant independent of the time-step size $\tau$. The estimate above implies an upper bound for $\left\|\mathcal{C}^{n}\right\|_{W^{1, \infty}}$ through the following discrete inhomogeneous Sobolev embedding:

$$
\left\|\mathcal{C}^{N}\right\|_{L^{\infty}\left(W^{1, \infty}\right)} \leq C\left(\left\|D_{\tau} \mathcal{C}^{N}\right\|_{L^{p}\left(L^{q}\right)}+\left\|\mathcal{C}^{N}\right\|_{L^{p}\left(W^{2, q}\right)}\right) \leq C_{p, q},
$$

which holds for sufficiently large $p$ and $q$ such that $\frac{2}{p}+\frac{d}{q}<1$.

By using the regularity estimate above, in Section 5. we further derive error estimate for the fully discrete solution in the $L^{p}\left(\widetilde{W}^{-1, q}\right)$ and $L^{p}\left(W^{1, q}\right)$ norm, i.e.,

$$
\left\|D_{\tau}\left(\mathcal{C}_{h}^{n}-\Pi_{h} \mathcal{C}^{n}\right)\right\|_{L^{p}\left(\widetilde{W}^{-1, q}\right)}+\left\|\mathcal{C}_{h}^{n}-\Pi_{h} \mathcal{C}^{n}\right\|_{L^{p}\left(W^{1, q}\right)} \leq C h,
$$

which yields an error estimate in $L^{\infty}\left(L^{\infty}\right)$ through the discrete inhomogeneous Sobolev embedding

$$
\left\|\mathcal{C}_{h}^{n}-\Pi_{h} \mathcal{C}^{n}\right\|_{L^{\infty}\left(L^{\infty}\right)} \leq C\left(\left\|D_{\tau}\left(\mathcal{C}_{h}^{n}-\Pi_{h} \mathcal{C}^{n}\right)\right\|_{L^{p}\left(\widetilde{W}^{-1, q}\right)}+\left\|\mathcal{C}_{h}^{n}-\Pi_{h} \mathcal{C}^{n}\right\|_{L^{p}\left(W^{1, q}\right)}\right) \leq C h
$$

for sufficiently large $p$ and $q$ such that $\frac{2}{p}+\frac{d}{q}<1$. By using the inverse inequality of the finite element space, we further obtain

$$
\left\|\mathcal{C}_{h}^{n}-\Pi_{h} \mathcal{C}^{n}\right\|_{L^{\infty}\left(W^{1, \infty}\right)} \leq C h^{-1}\left\|\mathcal{C}_{h}^{n}-\Pi_{h} \mathcal{C}^{n}\right\|_{L^{\infty}\left(L^{\infty}\right)} \leq C
$$

which implies upper bound for $\left\|\mathcal{C}_{h}^{n}\right\|_{W^{1, \infty}}$.

Throughout we denote $C_{p_{1}, \ldots, p_{k}}$ a generic positive constant which may be different at different occurrence, independent of $n, \tau$ and $h$, while possibly depend upon $K, T, \Omega$ and the parameters $p_{1}, \ldots, p_{k}$ in the subscript.

\section{Preliminaries}

In this section we introduce some notations and lemmas to be used in our proof of Theorem 2.1] The basic ideas for proving these lemmas are described, and the detailed proof can be found in Appendix.

We define a Ritz operator $\mathbf{R}_{h}(t): H^{1} \rightarrow S_{h}^{1}$ and an $L^{2}$-projection operator $\mathbf{P}_{h}^{r}: L^{2} \rightarrow S_{h}^{r}$ by

$$
\left(D(\mathbf{u}(\cdot, t)) \nabla\left(\phi-\mathbf{R}_{h} \phi\right), \nabla \varphi_{h}\right)+\left(\phi-\mathbf{R}_{h} \phi, \varphi_{h}\right)=0, \quad \forall \phi \in H^{1}, \quad \forall \varphi_{h} \in S_{h}^{1},
$$

and

$$
\left(\phi-\mathbf{P}_{h}^{r} \phi, \varphi_{h}\right)=0, \quad \forall \phi \in L^{2}, \forall \varphi_{h} \in S_{h}^{r},
$$

respectively, with the abbreviations $\mathbf{P}_{h}:=\mathbf{P}_{h}^{1}$ and $\overline{\mathbf{P}}_{h}:=\mathbf{P}_{h}^{2}$, which satisfy the following estimates:

$$
\begin{array}{ll}
\left\|\varphi-\mathbf{P}_{h}^{r} \varphi\right\|_{W^{\ell_{0}, q}} \leq C h^{m-\ell_{0}}\|\varphi\|_{W^{m, q}}, & \forall \varphi \in W^{m, q}, \\
\left\|\varphi-\mathbf{R}_{h} \varphi\right\|_{L^{s}}+h\left\|\varphi-\mathbf{R}_{h} \varphi\right\|_{W^{1, s}} \leq C h^{l}\|\varphi\|_{W^{l, s}}, & \forall \varphi \in W^{l, s}, \\
\left\|\varphi-\mathbf{R}_{h} \varphi\right\|_{L^{s}} \leq C h\left\|\varphi-\mathbf{R}_{h} \varphi\right\|_{W^{1, s}}, & \forall \varphi \in W^{l, s},
\end{array}
$$


for $\ell_{0}=0,1, \ell_{0} \leq m \leq r+1,1 \leq l \leq r+1,1 \leq q \leq \infty$ and $1<s<\infty$. Similarly, the Lagrangian interpolation operator $\Pi_{h}: C(\bar{\Omega}) \rightarrow S_{h}^{1}$ satisfies

$$
\left\|\Pi_{h} \varphi-\varphi\right\|_{L^{q}}+h\left\|\nabla\left(\Pi_{h} \varphi-\varphi\right)\right\|_{L^{q}} \leq C h^{2}\|\varphi\|_{W^{2, q}}, \quad \forall \varphi \in W^{2, q}, \quad \forall q \in[2, \infty) .
$$

For the system (1.9), we define a corresponding time-discrete (spatially continuous) system

$$
\begin{cases}D_{\tau} \Phi^{n}-\nabla \cdot\left(a\left(\cdot, t_{n}\right) \nabla \Phi^{n}\right)+\Phi^{n}=f^{n}-\nabla \cdot \mathbf{g}^{n} & \text { in } \Omega, \\ a\left(\cdot, t_{n}\right) \nabla \Phi^{n} \cdot \mathbf{n}=\mathbf{g}^{n} \cdot \mathbf{n} & \text { on } \partial \Omega, \\ \Phi^{0}=\phi_{0}(x) & \text { for } x \in \Omega,\end{cases}
$$

and a fully-discrete finite element system of $\Phi_{h}^{n} \in S_{h}^{r}, n=1,2, \ldots$,

$$
\left(D_{\tau} \Phi_{h}^{n}, v_{h}\right)+\left(a\left(\cdot, t_{n}\right) \nabla \Phi_{h}^{n}, \nabla v_{h}\right)+\left(\Phi_{h}^{n}, v_{h}\right)=\left(f^{n}, v_{h}\right)+\left(\mathbf{g}^{n}, \nabla v_{h}\right), \quad \forall v_{h} \in S_{h}^{r},
$$

where $f^{n}=f\left(\cdot, t_{n}\right)$ and $\mathbf{g}^{n}=\mathbf{g}\left(\cdot, t_{n}\right)$. Some existing estimates for the solutions of (3.5) and (3.6) are given in the following two lemmas.

Lemma 3.1. If the coefficient matrix $a(x, t)=\left(a_{i j}(x, t)\right)_{d \times d}$ in (3.5) and (3.6) satisfies

$$
\begin{aligned}
& \lambda^{-1} \sum_{i=1}^{d}\left|\xi_{i}\right|^{2} \leq \sum_{i, j=1}^{d} a_{i j}(x, t) \xi_{i} \xi_{j} \leq \lambda \sum_{i=1}^{d}\left|\xi_{i}\right|^{2}, \quad \forall \xi_{i} \in \mathbb{R}, \quad \forall(x, t) \in \Omega \times[0, T], \\
& a_{i j} \in L^{\infty}\left(0, T ; W^{1, \infty}(\Omega)\right) \quad \text { and } \quad \partial_{t} a_{i j} \in L^{\infty}\left(0, T ; L^{\infty}(\Omega)\right),
\end{aligned}
$$

then the time-discrete solutions defined by (3.5) satisfy

$$
\begin{aligned}
& \left\|D_{\tau} \Phi^{n}\right\|_{L^{p}\left(\widetilde{W}^{-1, q)}\right.}+\left\|\Phi^{n}\right\|_{L^{p}\left(W^{1, q}\right)} \leq C\left(\left\|f^{n}\right\|_{L^{p}\left(L^{q}\right)}+\left\|\mathbf{g}^{n}\right\|_{L^{p}\left(L^{q}\right)}\right), \quad \forall p, q \in(1, \infty), \\
& \left\|D_{\tau} \Phi^{n}\right\|_{L^{p}\left(L^{q}\right)}+\left\|\Phi^{n}\right\|_{L^{p}\left(W^{2, q}\right)} \leq C\left\|f^{n}\right\|_{L^{p}\left(L^{q}\right)}, \quad \text { if } \mathbf{g}=\mathbf{0}, \quad \forall p, q \in(1, \infty)
\end{aligned}
$$

The proof of (3.10) was given in [4] (also see [23, Theorem 3.1]) and the proof for (3.9) can be found in [32]. The following lemma is a consequence of [32, (1.18) and (2.3)-(2.4)].

Lemma 3.2. Let $\phi^{n}=\phi\left(\cdot, t_{n}\right), \Phi^{n}$ and $\Phi_{h}^{n}$ denote the solutions of (1.9), (3.5) and (3.6), respectively. Under the assumption of Lemma 3.1, there exist positive constants $\tau_{2}$ and $h_{2}$ such that the following estimates hold for $\tau \leq \tau_{2}, h \leq h_{2}$ and $p, q \in(1, \infty)$ :

$$
\begin{aligned}
& \left\|D_{\tau} \Phi_{h}^{n}\right\|_{L^{p}\left(\widetilde{W}^{-1, q}\right)}+\left\|\Phi_{h}^{n}\right\|_{L^{p}\left(W^{1, q}\right)} \leq C\left(\left\|f^{n}\right\|_{L^{p}\left(L^{q}\right)}+\left\|\mathbf{g}^{n}\right\|_{L^{p}\left(L^{q}\right)}\right), \\
& \left\|\mathbf{P}_{h} \phi^{n}-\Phi_{h}^{n}\right\|_{L^{p}\left(L^{q}\right)} \\
& \quad \leq C\left(\left\|\phi^{n}-\mathbf{R}_{h} \phi^{n}\right\|_{L^{p}\left(L^{q}\right)}+\left\|\mathbf{P}_{h} \phi_{0}(x)-\Phi_{h}^{0}\right\|_{L^{q}}+\tau\left\|\partial_{t t} \phi\right\|_{L^{p}\left(0, T ; \widetilde{W}^{-1, q}\right)}\right), \\
& \left\|D_{\tau}\left(\mathbf{P}_{h} \Phi^{n}-\Phi_{h}^{n}\right)\right\|_{L^{p}\left(\widetilde{W}^{-1, q}\right)}+\left\|\mathbf{P}_{h} \Phi^{n}-\Phi_{h}^{n}\right\|_{L^{p}\left(W^{1, q}\right)} \\
& \quad \leq C\left\|\Phi^{n}-\mathbf{R}_{h} \Phi^{n}\right\|_{L^{p}\left(W^{1, q}\right)}+C h^{-1}\left\|\mathbf{P}_{h} \Phi^{0}-\Phi_{h}^{0}\right\|_{L^{q}} .
\end{aligned}
$$

The estimates (3.11) and (3.12) can be found in [32, (1.18)] and [32, (2.4)], respectively, and (3.13) can be proved by using [32, (2.3)].

In addition, for the elliptic boundary value problem

$$
\begin{cases}\nabla \cdot(a \nabla u)=f+\nabla \cdot \mathbf{g} & \text { in } \Omega, \\ a \nabla u \cdot \mathbf{n}=\mathbf{g} \cdot \mathbf{n} & \text { on } \partial \Omega,\end{cases}
$$

with the constraint $\int_{\Omega} u \mathrm{~d} x=0$, the following $W^{2, q}$ and $C^{2, \alpha}$ estimates are consequences of [18, Theorem 2.4.2.7] and [35, Theorem 4.40 and Corollary 4.41].

Lemma 3.3. Assume that $\mathbf{g}=0, f \in L^{q}$ with $q \in[2, \infty)$ and $\int_{\Omega} f \mathrm{~d} x=0$, and the matrix $a=\left(a_{i j}\right)_{d \times d}$ satisfies the ellipticity condition (3.7).

(1) If $a_{i j} \in W^{1, \infty}$, then (3.14) has a unique solution $u \in W^{2, q}$ satisfying

$$
\|u\|_{W^{2, q}} \leq C_{q}\|f\|_{L^{q}}
$$

where the constant $C_{q}$ may depend on $\sum_{i, j=1}^{d}\left\|a_{i j}\right\|_{W^{1, \infty}}$. 
(2) If $a_{i j} \in C^{1, \alpha}$, then (3.14) has a unique solution $u \in C^{2, \alpha}$ satisfying

$$
\|u\|_{C^{2, \alpha}} \leq C\|f\|_{C^{\alpha}}
$$

where the constant $C$ may depend on $\alpha$ and $\sum_{i, j=1}^{d}\left\|a_{i j}\right\|_{C^{1, \alpha}}$.

Moreover, we need the following $C^{1, \alpha}$ estimate, which is a consequence of the steady-state case of the estimate in [34, Theorem 4.30].

Lemma 3.4. Assume that $f \in L^{\infty}, \mathbf{g} \in C^{\alpha}$ for a given $\alpha \in(0,1)$, and $a_{i j} \in C^{\alpha}$ satisfies the ellipticity condition (3.7). Then the solution of (3.14) satisfies

$$
\|u\|_{C^{1, \alpha}} \leq C\left(\|f\|_{L^{\infty}}+\|\mathbf{g}\|_{C^{\alpha}}\right)
$$

where the constant $C$ may depend on $\alpha$ and $\sum_{i, j=1}^{d}\left\|a_{i j}\right\|_{C^{\alpha}}$.

A $W^{1, q}$ estimate of the corresponding finite element solution is given in the following lemma (a consequence of [17, Corollary A.6]).

Lemma 3.5 ( $W^{1, q}$ estimate of elliptic finite element equations). Let $r \geq 1, q \in[2, \infty)$, and $\mathbf{g} \in\left(L^{q}\right)^{d}$. If the matrix $a=\left(a_{i j}\right)_{d \times d} \in W^{1, \infty}$ satisfies the ellipticity condition (3.7), then the finite element system

$$
\left(a \nabla u_{h}, \nabla v_{h}\right)=\left(\mathbf{g}, \nabla v_{h}\right), \quad \forall v_{h} \in \stackrel{\circ}{S}_{h}^{r},
$$

has a unique solution $u_{h} \in \stackrel{\circ}{S}_{h}^{r}$, satisfying

$$
\left\|u_{h}\right\|_{W^{1, q}} \leq C_{q}\|\mathbf{g}\|_{L^{q}},
$$

where $C_{q}$ may depend on $\sum_{i, j=1}^{d}\left\|a_{i j}\right\|_{W^{1, \infty}}$.

The following discrete version of inhomogeneous Sobolev embedding (as a consequence of 38 , Proposition 1.2.10]) establishes a connection between Lemmas 3.1]3.2 and the $L^{\infty}$ boundedness of numerical solutions.

Lemma 3.6 (Discrete inhomogeneous Sobolev embedding). Let $p, q \in(1, \infty)$ satisfy $2 / p+d / q<$ 1 , and let $\phi^{n} \in W^{1, q}, n=0,1,2, \ldots$, be a sequence of functions such that $\phi^{0}=0$. Then for $\alpha \in(0,1-2 / p-d / q)$ there holds

$$
\begin{aligned}
& \left\|\phi^{n}\right\|_{L^{\infty}\left(L^{\infty}\right)}+\left\|\phi^{n}\right\|_{L^{\infty}\left(C^{\alpha}\right)} \leq C\left(\left\|D_{\tau} \phi^{n}\right\|_{L^{p}\left(\widetilde{W}^{-1, q}\right)}+\left\|\phi^{n}\right\|_{L^{p}\left(W^{1, q}\right)}\right), \\
& \left\|\phi^{n}\right\|_{L^{\infty}\left(W^{1, \infty}\right)}+\left\|\phi^{n}\right\|_{L^{\infty}\left(C^{1, \alpha}\right)} \leq C\left(\left\|D_{\tau} \phi^{n}\right\|_{L^{p}\left(L^{q}\right)}+\left\|\phi^{n}\right\|_{L^{p}\left(W^{2, q}\right)}\right),
\end{aligned}
$$

where the constant $C$ is independent of $n \geq 1$.

The following lemma is an extension of the generalized Grönwall's inequality [31, Lemma 3.2] to the time-discrete setting.

Lemma 3.7. Let $1<p<\infty$ and let $Y^{n} \geq 0, n=0,1, \ldots, N$, be a sequence of numbers such that

$$
\left(\tau \sum_{n=k+1}^{m}\left|Y^{n}\right|^{p}\right)^{\frac{1}{p}} \leq \alpha\left(Y^{k}+\tau \sum_{n=k+1}^{m} Y^{n}\right)+\beta, \quad \forall 0 \leq k<m \leq N,
$$

for some positive constants $\alpha$ and $\beta$. Then there exists $\tau_{p}$ such that for $\tau \leq \tau_{p}$,

$$
\left(\tau \sum_{n=0}^{N}\left|Y^{n}\right|^{p}\right)^{\frac{1}{p}} \leq C_{T, \alpha, p}\left(Y^{0}+\beta\right)
$$

where the constants $\tau_{p}$ and $C_{T, \alpha, p}$ are independent of $\tau, \beta$ and the sequence $Y^{n}, n=0,1, \ldots, N$.

Besides the lemmas above, the following interpolation inequality will be frequently used:

$$
\|v\|_{L^{s}} \leq C_{\epsilon}\|v\|_{L^{s_{1}}}+\epsilon\|v\|_{L^{s_{2}}}, \quad \forall s \in\left(s_{1}, s_{2}\right),
$$

where $\epsilon>0$ can be arbitrarily small at the expense of enlarging the constant $C_{\epsilon}$. Since $W^{1, q} \hookrightarrow L^{\infty}$, it follows that

$$
\|v\|_{L^{s}} \leq C \epsilon\|v\|_{L^{2}}+\epsilon\|v\|_{L^{\infty}} \leq C_{\epsilon}\|v\|_{L^{2}}+\epsilon\|v\|_{W^{1, q}}, \quad \forall s \in(2, \infty) .
$$




\section{4. $L^{p}$ estimates for a time-discrete system}

We define a time-discrete system corresponding to (1.1)-(1.5) by

$$
\begin{aligned}
& -\nabla \cdot\left(\frac{k(x)}{\mu\left(\mathcal{C}^{n-1}\right)} \nabla P^{n-1}\right)=q_{I}^{n-1}-q_{P}^{n-1}, \quad n=1, \ldots, N+1, \\
& \gamma D_{\tau} \mathcal{C}^{n}-\nabla \cdot\left(D\left(\mathbf{U}^{n-1}\right) \nabla \mathcal{C}^{n}\right)+\mathcal{C}^{n} \\
& =\hat{c} q_{I}^{n}+\left(1-\frac{1}{2}\left(q_{I}^{n}+q_{P}^{n}\right)\right) \mathcal{C}^{n}-\frac{1}{2} \mathbf{U}^{n-1} \cdot \nabla \mathcal{C}^{n}-\frac{1}{2} \nabla \cdot\left(\mathbf{U}^{n-1} \mathcal{C}^{n}\right), \quad n=1, \ldots, N,
\end{aligned}
$$

with the boundary and initial conditions

$$
\begin{aligned}
& D\left(\mathbf{U}^{n-1}\right) \nabla \mathcal{C}^{n} \cdot \mathbf{n}=0, \quad \frac{k(x)}{\mu\left(\mathcal{C}^{n-1}\right)} \nabla P^{n-1} \cdot \mathbf{n}=0, \quad \text { for } x \in \partial \Omega, \\
& \mathcal{C}^{0}=c_{0}(x), \quad \text { for } x \in \Omega,
\end{aligned}
$$

where

$$
\mathbf{U}^{n-1}=-\frac{k(x)}{\mu\left(\mathcal{C}^{n-1}\right)} \nabla P^{n-1},
$$

and the condition $\int_{\Omega} P^{n-1} d x=0$ is enforced for the uniqueness of the solution of (4.1).

The fully discrete system (2.1)-(2.3) can be viewed as the spatial discretization of (4.1)-(4.5) by the FEM with P2 and P1 elements for $P^{n-1}$ and $\mathcal{C}^{n}$, respectively. The main result of this section is the following lemma on the $L^{p}$ and $L^{\infty}$ estimates for the time-discrete system (4.1)-(4.5). These estimates are needed for analyzing the fully discrete finite element solutions in the next section.

Lemma 4.1. Suppose that (1.1)-(1.5) has a unique solution satisfying (2.4) for some $q \in(d, \infty)$, and let $p \in(2, \infty)$ satisfy $2 / p+d / q<1$. Then the time-discrete system (4.1) -(4.5) has a unique solution $\left(P^{n}, \mathcal{C}^{n}\right) \in W^{2, q} \times W^{2, q}, n=0,1, \ldots, N$, such that

$$
\begin{aligned}
& \left\|D_{\tau} \mathcal{C}^{N}\right\|_{L^{p}\left(L^{q}\right)}+\left\|\mathcal{C}^{N}\right\|_{L^{p}\left(W^{2, q}\right)} \leq C_{p, q}, \\
& \left\|P^{n}\right\|_{W^{2, \infty}}+\left\|\mathbf{U}^{n}\right\|_{W^{1, \infty}}+\left\|D_{\tau} \mathbf{U}^{n}\right\|_{L^{\infty}}+\left\|\mathcal{C}^{n}\right\|_{W^{1, \infty}} \leq C_{p, q} .
\end{aligned}
$$

Proof. For a given $\mathcal{C}^{n-1} \in W^{2, q} \hookrightarrow C^{1, \alpha}$, with $\alpha=1-d / q \in(0,1)$, we have $\frac{k}{\mu\left(\mathcal{C}^{n-1}\right)} \in C^{1, \alpha}$. Then, by Lemma 3.3, (4.1) has a unique solution $P^{n-1} \in C^{2, \alpha} \hookrightarrow W^{2, \infty}$ such that

$$
\left\|P^{n-1}\right\|_{C^{2, \alpha}} \leq C_{\left\|\mathcal{C}^{n-1}\right\|_{C^{1, \alpha}}} .
$$

where $C_{\left\|\mathcal{C}^{n-1}\right\|_{C^{1, \alpha}}}$ is a constant depending on $\left\|\mathcal{C}^{n-1}\right\|_{C^{1, \alpha}}$. In view of (4.5), $\mathbf{U}^{n-1} \in C^{1, \alpha} \hookrightarrow W^{1, \infty}$, i.e.,

$$
\left\|\mathbf{U}^{n-1}\right\|_{C^{1, \alpha}} \leq C_{\left\|\mathcal{C}^{n-1}\right\|_{C^{1, \alpha}}} .
$$

Thus by [18, Theorem 2.4.2.7], the elliptic equation (4.2) has a unique solution $\mathcal{C}^{n} \in W^{2, q}$, i.e.,

$$
\left\|\mathcal{C}^{n}\right\|_{W^{2, q}} \leq C_{\left\|\mathbf{U}^{n-1}\right\|_{C^{1, \alpha}}} \leq C_{\left\|\mathcal{C}^{n-1}\right\|_{C^{1, \alpha}}} \leq C_{\left\|\mathcal{C}^{n-1}\right\|_{W^{2, q}}}
$$

This proves the existence and uniqueness of solutions $\left(P^{n}, \mathcal{C}^{n}\right) \in W^{2, q} \times W^{2, q}, n=0,1, \ldots, N$. In particular, there exists an increasing function $\varphi: \mathbb{R}_{+} \rightarrow \mathbb{R}_{+}$such that $\varphi(s) \geq s$ and

$$
\left\|\mathcal{C}^{n}\right\|_{W^{2, q}}+\left\|P^{n}\right\|_{C^{2, \alpha}}+\left\|\mathbf{U}^{n}\right\|_{C^{1, \alpha}} \leq \varphi\left(\left\|\mathcal{C}^{n-1}\right\|_{W^{2, q}}\right)
$$

It remains to prove the quantitative regularity estimate (4.6)-(4.7). To simplify the notations, we omit the dependence on $p$ and $q$ in the subscripts of the generic constant $C$.

We start with proving the following suboptimal $L^{\infty}$ error estimate by mathematical induction:

$$
\left\|\mathbf{u}^{n}-\mathbf{U}^{n}\right\|_{L^{\infty}}+\left\|c^{n}-\mathcal{C}^{n}\right\|_{L^{\infty}} \leq \tau^{1 / 2} .
$$

Since $c^{0}-\mathcal{C}^{0}=0$, the inequality above holds for $n=0$. We assume that (4.12) holds for $0 \leq n \leq$ $m-1$ and below, we prove that it also holds for $n=m$.

From (1.2) and (4.1), we see that

$$
\nabla \cdot\left(\frac{k(x)}{\mu\left(c^{n-1}\right)} \nabla\left(p^{n-1}-P^{n-1}\right)\right)=\nabla \cdot\left(\left(\frac{k(x)}{\mu\left(c^{n-1}\right)}-\frac{k(x)}{\mu\left(\mathcal{C}^{n-1}\right)}\right) \nabla\left(p^{n-1}-P^{n-1}\right)\right)
$$




$$
+\nabla \cdot\left(\left(\frac{k(x)}{\mu\left(\mathcal{C}^{n-1}\right)}-\frac{k(x)}{\mu\left(c^{n-1}\right)}\right) \nabla p^{n-1}\right) .
$$

By the $W^{1, q}$ estimate of elliptic equations (see [5, Theorem 1]), we get

$$
\begin{aligned}
& \left\|p^{n-1}-P^{n-1}\right\|_{W^{1, q}} \\
& \leq C\left\|\left(\frac{k(x)}{\mu\left(c^{n-1}\right)}-\frac{k(x)}{\mu\left(\mathcal{C}^{n-1}\right)}\right) \nabla\left(p^{n-1}-P^{n-1}\right)\right\|_{L^{q}}+C_{q}\left\|\left(\frac{k(x)}{\mu\left(\mathcal{C}^{n-1}\right)}-\frac{k(x)}{\mu\left(c^{n-1}\right)}\right) \nabla p^{n-1}\right\|_{L^{q}} \\
& \leq C_{q}\left\|c^{n-1}-\mathcal{C}^{n-1}\right\|_{L^{\infty}}\left\|\nabla\left(p^{n-1}-P^{n-1}\right)\right\|_{L^{q}}+C_{q}\left\|c^{n-1}-\mathcal{C}^{n-1}\right\|_{L^{q}}\left\|\nabla p^{n-1}\right\|_{L^{\infty}} \\
& \leq C_{q} \tau^{\frac{1}{2}}\left\|p^{n-1}-P^{n-1}\right\|_{W^{1, q}}+C_{q}\left\|c^{n-1}-\mathcal{C}^{n-1}\right\|_{L^{q}}, \quad \text { for } n=1, \ldots, m,
\end{aligned}
$$

where we have used the induction assumption (4.12) in the last inequality. When $\tau \leq \tau_{1}$ for some $\tau_{1}>0$, the last inequality further implies

$$
\left\|p^{n-1}-P^{n-1}\right\|_{W^{1, q}} \leq C_{q}\left\|c^{n-1}-\mathcal{C}^{n-1}\right\|_{L^{q}}, \quad \text { for } n=1, \ldots, m .
$$

By using (1.2) and (4.5), we have

$$
\begin{aligned}
\left\|\mathbf{u}^{n}-\mathbf{U}^{n}\right\|_{L^{s}} & =\left\|-\left(\frac{k(x)}{\mu\left(c^{n}\right)}-\frac{k(x)}{\mu\left(\mathcal{C}^{n}\right)}\right) \nabla p^{n}-\frac{k(x)}{\mu\left(\mathcal{C}^{n}\right)} \nabla\left(p^{n}-P^{n}\right)\right\|_{L^{s}} \\
& \leq C\left\|c^{n}-\mathcal{C}^{n}\right\|_{L^{s}}\left\|\nabla p^{n}\right\|_{L^{\infty}}+C\left\|p^{n}-P^{n}\right\|_{W^{1, s}} \\
& \leq C\left\|c^{n}-\mathcal{C}^{n}\right\|_{L^{s}}+C\left\|p^{n}-P^{n}\right\|_{W^{1, s}}, \quad \text { for } n=0,1, \ldots, m,
\end{aligned}
$$

for any $s \in[1, \infty]$.

We rewrite (1.1) into

where

$$
\begin{aligned}
& \gamma D_{\tau} c^{n}-\nabla \cdot\left(D\left(\mathbf{u}^{n-1}\right) \nabla c^{n}\right)+c^{n} \\
& =\widehat{c} q_{I}^{n}+\left(1-\frac{1}{2}\left(q_{I}^{n}+q_{P}^{n}\right)\right) c^{n}-\frac{1}{2} \mathbf{u}^{n-1} \cdot \nabla c^{n}-\frac{1}{2} \nabla \cdot\left(\mathbf{u}^{n-1} c^{n}\right)+E_{t r}^{n}
\end{aligned}
$$

$$
\begin{aligned}
E_{t r}^{n}= & \gamma D_{\tau} c^{n}-\gamma c_{t}^{n}+\nabla \cdot\left(\left(D\left(\mathbf{u}^{n}\right)-D\left(\mathbf{u}^{n-1}\right)\right) \nabla c^{n}\right)+\left(\mathbf{u}^{n-1}-\mathbf{u}^{n}\right) \cdot \nabla c^{n} \\
& -\frac{1}{2}\left(\left(q_{I}^{n}-q_{P}^{n}\right)-\left(q_{I}^{n-1}-q_{P}^{n-1}\right)\right) c^{n}
\end{aligned}
$$

denotes the truncation error, satisfying the following estimate under the regularity assumption (2.4):

Subtracting (4.2) from (4.16) gives

$$
\left\|E_{t r}^{n}\right\|_{L^{p}\left(\widetilde{W}^{-1, q}\right)} \leq C \tau .
$$

$$
\begin{aligned}
& \gamma D_{\tau}\left(c^{n}-\mathcal{C}^{n}\right)-\nabla \cdot\left(D\left(\mathbf{u}^{n-1}\right) \nabla\left(c^{n}-\mathcal{C}^{n}\right)\right)+c^{n}-\mathcal{C}^{n} \\
= & \left(1-\frac{1}{2}\left(q_{I}^{n}+q_{P}^{n}\right)\right)\left(c^{n}-\mathcal{C}^{n}\right)+\frac{1}{2}\left(q_{I}^{n-1}-q_{P}^{n-1}\right)\left(c^{n}-\mathcal{C}^{n}\right)-\frac{1}{2}\left(\mathbf{u}^{n-1}-\mathbf{U}^{n-1}\right) \cdot \nabla c^{n} \\
& -\frac{1}{2} \nabla \cdot\left(\mathbf{U}^{n-1}\left(c^{n}-\mathcal{C}^{n}\right)\right)-\frac{1}{2} \nabla \cdot\left(\left(\mathbf{u}^{n-1}-\mathbf{U}^{n-1}\right) c^{n}+\mathbf{U}^{n-1}\left(c^{n}-\mathcal{C}^{n}\right)\right) \\
& +\nabla \cdot\left(\left(D\left(\mathbf{u}^{n-1}\right)-D\left(\mathbf{U}^{n-1}\right)\right) \nabla\left(\mathcal{C}^{n}-c^{n}\right)\right)+\nabla \cdot\left(\left(D\left(\mathbf{u}^{n-1}\right)-D\left(\mathbf{U}^{n-1}\right)\right) \nabla c^{n}\right)+E_{t r}^{n} .
\end{aligned}
$$

Applying Lemma 3.1 to the last equation yields, for $p \in(2, \infty)$ and $n=1, \ldots, m$,

$$
\begin{aligned}
& \left\|D_{\tau}\left(c^{n}-\mathcal{C}^{n}\right)\right\|_{L^{p}\left(\widetilde{W}^{-1, q}\right)}+\left\|c^{n}-\mathcal{C}^{n}\right\|_{L^{p}\left(W^{1, q}\right)} \\
& \leq C \\
& \quad+\left(1-\frac{1}{2}\left(q_{I}^{n}+q_{P}^{n}\right)\right)\left(c^{n}-\mathcal{C}^{n}\right)\left\|_{L^{p}\left(L^{q}\right)}+C\right\|\left(q_{I}^{n-1}-q_{P}^{n-1}\right)\left(c^{n}-\mathcal{C}^{n}\right) \|_{L^{p}\left(L^{q}\right)} \\
& \quad+C\left\|\mathbf{u}^{n-1}-\mathbf{U}^{n-1}\right\|_{L^{p}\left(L^{q}\right)}\left\|\nabla c^{n}\right\|_{L^{\infty}\left(L^{\infty}\right)}+C\left\|\mathbf{U}^{n-1}\right\|_{L^{\infty}\left(L^{\infty}\right)}\left\|c^{n}-\mathcal{C}^{n}\right\|_{L^{p}\left(L^{q}\right)} \\
& \quad+C\left\|\mathbf{u}^{n-1}-\mathbf{U}^{n-1}\right\|_{L^{p}\left(L^{q}\right)}\left\|c^{n}\right\|_{L^{\infty}\left(L^{\infty}\right)}+C\left\|\mathbf{U}^{n-1}\right\|_{L^{\infty}\left(L^{\infty}\right)}\left\|c^{n}-\mathcal{C}^{n}\right\|_{L^{p}\left(L^{q}\right)} \\
& \quad+C\left\|D\left(\mathbf{u}^{n-1}\right)-D\left(\mathbf{U}^{n-1}\right)\right\|_{L^{\infty}\left(L^{\infty}\right)}\left\|\nabla\left(\mathcal{C}^{n}-c^{n}\right)\right\|_{L^{p}\left(L^{q}\right)} \\
& \quad+C\left\|D\left(\mathbf{u}^{n-1}\right)-D\left(\mathbf{U}^{n-1}\right)\right\|_{L^{p}\left(L^{q}\right)}\left\|\nabla c^{n}\right\|_{L^{\infty}\left(L^{\infty}\right)}+C\left\|E_{t r}^{n}\right\|_{L^{p}\left(\widetilde{W}^{-1, q}\right)} \\
& \leq C\left(\left\|c^{n-1}-\mathcal{C}^{n-1}\right\|_{L^{p}\left(L^{q}\right)}+\left\|c^{n}-\mathcal{C}^{n}\right\|_{L^{p}\left(L^{q}\right)}\right)+C \tau^{1 / 2}\left\|c^{n}-\mathcal{C}^{n}\right\|_{L^{p}\left(W^{1, q}\right)}+C \tau \\
& \leq C\left\|c^{n}-\mathcal{C}^{n}\right\|_{L^{p}\left(L^{q}\right)}+C \tau^{1 / 2}\left\|c^{n}-\mathcal{C}^{n}\right\|_{L^{p}\left(W^{1, q}\right)}+C \tau,
\end{aligned}
$$


where we have used induction assumption (4.12) to estimate $\left\|D\left(\mathbf{u}^{n-1}\right)-D\left(\mathbf{U}^{n-1}\right)\right\|_{L^{\infty}\left(L^{\infty}\right)}$ and $\left\|\mathbf{U}^{n-1}\right\|_{L^{\infty}\left(L^{\infty}\right)}$, and used (4.14)-(4.15) to estimate $\left\|\mathbf{u}^{n-1}-\mathbf{U}^{n-1}\right\|_{L^{p}\left(L^{q}\right)}$. When $\tau \leq \tau_{2}$ for some $\tau_{2}>0$, the last inequality reduces to

$$
\left\|D_{\tau}\left(c^{n}-\mathcal{C}^{n}\right)\right\|_{L^{p}\left(\widetilde{W}^{-1, q)}\right.}+\left\|c^{n}-\mathcal{C}^{n}\right\|_{L^{p}\left(W^{1, q}\right)} \leq C\left\|c^{n}-\mathcal{C}^{n}\right\|_{L^{p}\left(L^{q}\right)}+C \tau, \quad n=1, \ldots, m .
$$

By Lemma 3.6,

$$
\begin{aligned}
\left\|c^{n}-\mathcal{C}^{n}\right\|_{L^{\infty}\left(L^{\infty}\right)} & \leq C\left(\left\|D_{\tau}\left(c^{n}-\mathcal{C}^{n}\right)\right\|_{L^{p}\left(\widetilde{W}^{-1, q}\right)}+\left\|c^{n}-\mathcal{C}^{n}\right\|_{L^{p}\left(W^{1, q}\right)}\right) \\
& \leq C\left\|c^{n}-\mathcal{C}^{n}\right\|_{L^{p}\left(L^{q}\right)}+C \tau \quad[\text { [4.19) is used here] } \\
& \leq C\left\|c^{n}-\mathcal{C}^{n}\right\|_{L^{p}\left(L^{\infty}\right)}+C \tau \\
& \leq \frac{1}{2}\left\|c^{n}-\mathcal{C}^{n}\right\|_{L^{\infty}\left(L^{\infty}\right)}+C_{p, q}\left\|c^{n}-\mathcal{C}^{n}\right\|_{L^{1}\left(L^{\infty}\right)}+C \tau, \quad n=1, \ldots, m,
\end{aligned}
$$

which further implies (through applying Gronwall's inequality, i.e., Lemma 3.7)

$$
\left\|c^{m}-\mathcal{C}^{m}\right\|_{L^{\infty}\left(L^{\infty}\right)} \leq C \tau \text {. }
$$

Substituting the inequality above into (4.19), we have

$$
\left\|D_{\tau}\left(c^{m}-\mathcal{C}^{m}\right)\right\|_{L^{p}\left(\widetilde{W}^{-1, q}\right)}+\left\|c^{m}-\mathcal{C}^{m}\right\|_{L^{p}\left(W^{1, q}\right)} \leq C \tau
$$

which with (3.20) shows

$$
\left\|c^{m}-\mathcal{C}^{m}\right\|_{L^{\infty}\left(C^{\alpha}\right)} \leq C\left(\left\|D_{\tau}\left(c^{m}-\mathcal{C}^{m}\right)\right\|_{L^{p}\left(\widetilde{W}^{-1, q}\right)}+\left\|c^{m}-\mathcal{C}^{m}\right\|_{L^{p}\left(W^{1, q}\right)}\right) \leq C \tau .
$$

By using an inverse inequality in time, (4.21) implies

$$
\left\|c^{m}-\mathcal{C}^{m}\right\|_{L^{\infty}\left(W^{1, q}\right)} \leq C \tau^{1-1 / p} .
$$

Moreover, applying (3.15) to (4.13) leads to, for $n=0,1, \ldots, m$,

$$
\begin{aligned}
& \left\|p^{n}-P^{n}\right\|_{W^{2, q}} \\
& \leq C\left\|\nabla \cdot\left(\left(\frac{k(x)}{\mu\left(c^{n}\right)}-\frac{k(x)}{\mu\left(\mathcal{C}^{n}\right)}\right) \nabla\left(p^{n}-P^{n}\right)\right)\right\|_{L^{q}}+C\left\|\nabla \cdot\left(\left(\frac{k(x)}{\mu\left(\mathcal{C}^{n}\right)}-\frac{k(x)}{\mu\left(c^{n}\right)}\right) \nabla p^{n}\right)\right\|_{L^{q}} \\
& \leq C\left(\left\|c^{n}-\mathcal{C}^{n}\right\|_{L^{\infty}}\left\|p^{n}-P^{n}\right\|_{W^{2, q}}+\left\|c^{n}-\mathcal{C}^{n}\right\|_{W^{1, q}}\left\|p^{n}-P^{n}\right\|_{W^{1, \infty}}\right) \\
& \quad+C\left(\left\|c^{n}-\mathcal{C}^{n}\right\|_{L^{\infty}}\left\|p^{n}\right\|_{W^{2, q}}+\left\|c^{n}-\mathcal{C}^{n}\right\|_{W^{1, q}}\left\|p^{n}\right\|_{W^{1, \infty}}\right) \\
& \leq C\left(\tau\left\|p^{n}-P^{n}\right\|_{W^{2, q}}+\tau^{1-1 / p}\left\|p^{n}-P^{n}\right\|_{W^{2, q}}\right)+C\left(\tau+\left\|c^{n}-\mathcal{C}^{n}\right\|_{W^{1, q}}\right),
\end{aligned}
$$

where we used (4.20)-(4.23) in deriving the last inequality. When $\tau \leq \tau_{3}$ for some $\tau_{3}>0$, we see that

$$
\left\|p^{n}-P^{n}\right\|_{W^{2, q}} \leq C\left(\tau+\left\|c^{n}-\mathcal{C}^{n}\right\|_{W^{1, q}}\right), \quad n=0,1, \ldots, m .
$$

By noting (4.21) and the Sobolev embedding $W^{2, q} \hookrightarrow W^{1, \infty}$ for $q>d$, we obtain

$$
\left\|p^{m}-P^{m}\right\|_{L^{p}\left(W^{1, \infty}\right)} \leq C\left\|p^{m}-P^{m}\right\|_{L^{p}\left(W^{2, q}\right)} \leq C\left(\tau+\left\|c^{m}-\mathcal{C}^{m}\right\|_{L^{p}\left(W^{1, q}\right)}\right) \leq C \tau
$$

which, together with an inverse inequality in time, leads to

$$
\left\|p^{m}-P^{m}\right\|_{L^{\infty}\left(W^{1, \infty}\right)}+\left\|p^{m}-P^{m}\right\|_{L^{\infty}\left(W^{2, q}\right)} \leq C \tau^{1-1 / p} .
$$

By taking $s=\infty$ in (4.15) and using (4.20), we get

$$
\left\|\mathbf{u}^{m}-\mathbf{U}^{m}\right\|_{L^{\infty}\left(L^{\infty}\right)} \leq C \tau^{1-1 / p} .
$$

Since $\frac{2}{p}+\frac{d}{q}<1$ implies $p>2$ and therefore $C \tau^{1-1 / p} \leq \tau^{1 / 2}$ for sufficiently small stepsize $\tau$, by combining above result and (4.20), the mathematical induction on (4.12) is closed as $\tau \leq \tau_{4}$ for some $\tau_{4}>0$. Consequently, the estimates (4.20), (4.23), (4.26) and (4.27) hold for $m=N$. When $\tau \leq \min _{1 \leq j \leq 4} \tau_{j}$, we have the following estimates:

$$
\left\|\mathcal{C}^{n}\right\|_{L^{\infty}}+\left\|\mathcal{C}^{n}\right\|_{W^{1, q}}+\left\|P^{n}\right\|_{W^{1, \infty}}+\left\|P^{n}\right\|_{W^{2, q}}+\left\|\mathbf{U}^{n}\right\|_{L^{\infty}}+\left\|D_{\tau} C^{n}\right\|_{L^{\infty}} \leq C .
$$

From (4.5) we further see that

$$
\left\|\mathbf{U}^{n}\right\|_{W^{1, q}} \leq C\left(\left\|P^{n}\right\|_{W^{2, q}}+\left\|\mathcal{C}^{n}\right\|_{W^{1, q}}\left\|P^{n}\right\|_{W^{1, \infty}}\right) \leq C, \quad n=0,1, \ldots, N .
$$


Now we are ready to prove (4.6)-(4.7). To prove (4.6), we rewrite (4.2) into

$$
\begin{aligned}
& \gamma D_{\tau} \mathcal{C}^{n}-\nabla \cdot\left(D\left(\mathbf{u}^{n-1}\right) \nabla \mathcal{C}^{n}\right)+\mathcal{C}^{n} \\
& =\hat{c} q_{I}^{n}+\left(1-\frac{1}{2}\left(q_{I}^{n}+q_{P}^{n}\right)\right) \mathcal{C}^{n}-\frac{1}{2} \mathbf{U}^{n-1} \cdot \nabla \mathcal{C}^{n}-\frac{1}{2} \nabla \cdot\left(\mathbf{U}^{n-1} \mathcal{C}^{n}\right) \\
& \quad+\nabla \cdot\left(\left(D\left(\mathbf{U}^{\mathbf{n}-\mathbf{1}}\right)-D\left(\mathbf{u}^{n-1}\right)\right) \nabla \mathcal{C}^{n}\right)
\end{aligned}
$$

and by Lemma 3.1, we obtain

$$
\begin{aligned}
& \left\|D_{\tau} \mathcal{C}^{N}\right\|_{L^{p}\left(L^{q}\right)}+\left\|\mathcal{C}^{N}\right\|_{L^{p}\left(W^{2, q}\right)} \\
& \leq C\left\|\hat{c} q_{I}^{N}\right\|_{L^{p}\left(L^{q}\right)}+C\left\|\left(1-\frac{1}{2}\left(q_{I}^{N}+q_{P}^{N}\right)\right) \mathcal{C}^{N}\right\|_{L^{p}\left(L^{q}\right)}+C\left\|\mathbf{U}^{N-1}\right\|_{L^{\infty}\left(L^{\infty}\right)}\left\|\nabla \mathcal{C}^{N}\right\|_{L^{p}\left(L^{q}\right)} \\
& \quad+C\left\|\nabla \cdot\left(\mathbf{U}^{N-1} \mathcal{C}^{N}\right)\right\|_{L^{p}\left(L^{q}\right)}+C\left\|\nabla \cdot\left(\left(D\left(\mathbf{U}^{N-1}\right)-D\left(\mathbf{u}^{N-1}\right)\right) \nabla \mathcal{C}^{N}\right)\right\|_{L^{p}\left(L^{q}\right)} \\
& \left.\leq C+C\left\|\nabla \cdot\left(\left(D\left(\mathbf{U}^{N-1}\right)-D\left(\mathbf{u}^{N-1}\right)\right) \nabla \mathcal{C}^{N}\right)\right\|_{L^{p}\left(L^{q}\right)}\right) \\
& \leq C+C\left(\left\|\nabla \mathbf{U}^{N-1}\right\|_{L^{\infty}\left(L^{q}\right)}+\left\|\nabla \mathbf{u}^{N-1}\right\|_{L^{\infty}\left(L^{q}\right)}\right)\left\|\nabla \mathcal{C}^{N}\right\|_{L^{p}\left(L^{\infty}\right)} \\
& \quad+C\left\|D\left(\mathbf{U}^{N-1}\right)-D\left(\mathbf{u}^{N-1}\right)\right\|_{L^{\infty}\left(L^{\infty}\right)}\left\|\mathcal{C}^{N}\right\|_{L^{p}\left(W^{2, q}\right)} \\
& \leq C+C\left\|\mathcal{C}^{N}\right\|_{L^{p}\left(W^{1, \infty}\right)}+C \tau^{\frac{1}{2}\left\|\mathcal{C}^{N}\right\|_{L^{p}\left(W^{2, q}\right)} .} \text { (4t.29)) }
\end{aligned}
$$

By noting $\left\|\mathcal{C}^{n}\right\|_{W^{1, \infty}} \leq \frac{1}{2}\left\|\mathcal{C}^{n}\right\|_{W^{2, q}}+C\left\|\mathcal{C}^{n}\right\|_{W^{1, q}}$ and (4.28), when $\tau \leq \tau_{5}$ for some $\tau_{5}>0$, (4.30) reduces to

$$
\left\|D_{\tau} \mathcal{C}^{N}\right\|_{L^{p}\left(L^{q}\right)}+\left\|\mathcal{C}^{N}\right\|_{L^{p}\left(W^{2, q}\right)} \leq C+C\left\|\mathcal{C}^{N}\right\|_{L^{p}\left(W^{1, q}\right)} \leq C
$$

(4.6) is obtained.

To prove (4.7), we use (4.31) and Lemma 3.6, which imply

$$
\left\|\mathcal{C}^{N}\right\|_{L^{\infty}\left(C^{1, \alpha}\right)} \leq C\left(\left\|D_{\tau} \mathcal{C}^{N}\right\|_{L^{p}\left(L^{q}\right)}+\left\|\mathcal{C}^{N}\right\|_{L^{p}\left(W^{2, q}\right)}\right) \leq C .
$$

With the regularity estimate above, applying [Lemma 3.3, (3.16)] to (4.1) yields

$$
\left\|P^{n}\right\|_{C^{2, \alpha}} \leq C\left\|q_{I}^{n}-q_{P}^{n}\right\|_{C^{\alpha}} \leq C, \quad n=0,1, \ldots, N,
$$

and substituting (4.32)-(4.33) into (4.5) gives

$$
\left\|\mathbf{U}^{n}\right\|_{C^{1, \alpha}} \leq C, \quad n=0,1, \ldots, N
$$

Again, applying the backward difference operator $D_{\tau}$ to (4.1) yields

$$
-\nabla \cdot\left(\frac{k(x)}{\mu\left(\mathcal{C}^{n}\right)} \nabla D_{\tau} P^{n}\right)-\nabla \cdot\left(D_{\tau}\left(\frac{k(x)}{\mu\left(\mathcal{C}^{n}\right)}\right) \nabla P^{n-1}\right)=D_{\tau} q_{I}^{n}-D_{\tau} q_{P}^{n} .
$$

By Lemma 3.4,

$$
\begin{aligned}
\left\|D_{\tau} P^{n}\right\|_{C^{1, \alpha}} & \leq \frac{C}{\tau}\left\|\left(\frac{k(x)}{\mu\left(\mathcal{C}^{n}\right)}-\frac{k(x)}{\mu\left(\mathcal{C}^{n-1}\right)}\right) \nabla P^{n-1}\right\|_{C^{\alpha}}+C\left\|D_{\tau} q_{I}^{n}-D_{\tau} q_{P}^{n}\right\|_{L^{\infty}} \\
& \leq \frac{C}{\tau}\left\|\mathcal{C}^{n}-\mathcal{C}^{n-1}\right\|_{C^{\alpha}}\left\|\nabla P^{n-1}\right\|_{C^{\alpha}}+C\left(\left\|\partial_{t} q_{I}\right\|_{L^{\infty}\left(0, T ; L^{\infty}\right)}+\left\|\partial_{t} q_{P}\right\|_{L^{\infty}\left(0, T ; L^{\infty}\right)}\right) \\
& \leq \frac{C}{\tau}\left\|c^{n}-c^{n-1}\right\|_{C^{\alpha}}+\frac{C}{\tau}\left\|\mathcal{C}^{n}-c^{n}\right\|_{C^{\alpha}}+\frac{C}{\tau}\left\|\mathcal{C}^{n-1}-c^{n-1}\right\|_{C^{\alpha}}+C \\
& \leq C, \quad n=1, \ldots, N,
\end{aligned}
$$

where we have used (4.22) in the last inequality. Finally, from (4.5) we see that

$$
\left\|D_{\tau} \mathbf{U}^{n}\right\|_{L^{\infty}} \leq C\left(\left\|\nabla D_{\tau} P^{n}\right\|_{L^{\infty}}+\left\|D_{\tau}\left(\frac{k(x)}{\mu\left(\mathcal{C}^{n}\right)}\right)\right\|_{L^{\infty}}\right) \leq C, \quad n=1, \ldots, N,
$$

and (4.7) follows immediately. This proves Lemma 4.1 in the case $\tau \leq \tau_{p, q}^{*}:=\min _{1 \leq j \leq 4} \tau_{j}$.

$$
\begin{aligned}
& \text { If } \tau \geq \tau_{p, q}^{*}, N=T / \tau \leq T / \tau_{p, q}^{*} \leq C \text {, and therefore, (4.11) implies } \\
& \qquad\left\|\mathcal{C}^{n}\right\|_{W^{2, q}}+\left\|P^{n}\right\|_{C^{2, \alpha}}+\left\|\mathbf{U}^{n}\right\|_{C^{1, \alpha}} \leq \varphi^{(n)}\left(\left\|\mathcal{C}^{0}\right\|_{W^{2, q}}\right) \leq \varphi^{\left(T / \tau_{p, q}^{*}\right)}\left(\left\|\mathcal{C}^{0}\right\|_{W^{2, q}}\right) \leq C,
\end{aligned}
$$

where $\varphi^{(n)}:=\varphi^{(n-1)} \circ \varphi$. This proves Lemma 4.1 in the case $\tau \geq \tau_{p, q}^{*}$.

\section{The proof of Theorem 2.1}

Before proving Theorem 2.1, we show the boundedness of the numerical solutions based on the uniform regularity estimates given in Lemma 4.1 for the time-discrete system (4.1)-(4.5). 


\subsection{Boundedness of the numerical solutions}

Lemma 5.1. Under the assumption of Theorem [2.1, there exist positive constants $\tau_{q}$ and $h_{q}$ such that for $\tau \leq \tau_{q}$ and $h \leq h_{q}$ the finite element system (2.1) -(2.3) has a unique solution $\left(P_{h}^{n}, \mathcal{C}_{h}^{n}\right)$, $n=0,1, \ldots, N$, satisfying the following estimates:

$$
\left\|\mathcal{C}_{h}^{n}\right\|_{W^{1, \infty}}+\left\|\mathbf{U}_{h}^{n}\right\|_{L^{\infty}} \leq C .
$$

Proof. Since both coefficient matrices of the linear systems (2.1) and (2.2) are positive definite (possibly non-symmetric), it follows that the linear system (2.1)-(2.2) has a unique solution.

Next, we prove a primary estimate

$$
\left\|\mathbf{P}_{h} \mathcal{C}^{n}-\mathcal{C}_{h}^{n}\right\|_{L^{\infty}} \leq h^{\frac{1}{2}}, \quad n=0, \ldots, m-1,
$$

by mathematical induction. For the given $q>d$, we choose a fixed $p \in(2, \infty)$ satisfying $2 / p+d / q<$ 1 , and omit the dependence on $p$ and $q$ in the subscripts of generic constants below.

Since $\left\|\mathbf{P}_{h} \mathcal{C}^{0}-\mathcal{C}_{h}^{0}\right\|_{L^{\infty}}=\left\|\mathbf{P}_{h} c_{0}-\Pi_{h} c_{0}\right\|_{L^{\infty}} \leq C h\left\|c_{0}\right\|_{W^{1, \infty}}$, (5.2) holds for $m=1$ when $h \leq h_{1}$ for some $h_{1}>0$. Therefore, we can assume that it holds for some positive integer $m$.

From (4.1), we see that

$$
\left(\frac{k(x)}{\mu\left(\mathcal{C}^{n-1}\right)} \nabla P^{n-1}, \nabla v_{h}\right)=\left(q_{I}^{n-1}-q_{P}^{n-1}, v_{h}\right), \quad \forall v_{h} \in \stackrel{S}{h}_{h}^{2} .
$$

and therefore, subtracting the equation above from (2.1) yields

$$
\begin{aligned}
\nabla \cdot\left(\frac{k(x)}{\mu\left(\mathcal{C}^{n-1}\right)} \nabla\left(\overline{\mathbf{P}}_{h} P^{n-1}-P_{h}^{n-1}\right)\right)= & \nabla \cdot\left(\left(\frac{k(x)}{\mu\left(\mathcal{C}^{n-1}\right)}-\frac{k(x)}{\mu\left(\mathcal{C}_{h}^{n-1}\right)}\right) \nabla\left(P^{n-1}-P_{h}^{n-1}\right)\right) \\
& +\nabla \cdot\left(\left(\frac{k(x)}{\mu\left(\mathcal{C}_{h}^{n-1}\right)}-\frac{k(x)}{\mu\left(\mathcal{C}^{n-1}\right)}\right) \nabla P^{n-1}\right) \\
& +\nabla \cdot\left(\frac{k(x)}{\mu\left(\mathcal{C}^{n-1}\right)} \nabla\left(\overline{\mathbf{P}}_{h} P^{n-1}-P^{n-1}\right)\right) .
\end{aligned}
$$

Since $\left\|\frac{k(x)}{\mu\left(\mathcal{C}^{n-1}\right)}\right\|_{W^{1, \infty}} \leq C$ (as a consequence of [Lemma 4.1, [4.7] ]), by the $W^{1, s}$ estimate of elliptic finite element system (Lemma 3.5), we have

$$
\begin{aligned}
& \left\|P^{n-1}-P_{h}^{n-1}\right\|_{W^{1, s}} \\
& \leq C\left\|\left(\frac{k(x)}{\mu\left(\mathcal{C}^{n-1}\right)}-\frac{k(x)}{\mu\left(\mathcal{C}_{h}^{n-1}\right)}\right) \nabla\left(P^{n-1}-P_{h}^{n-1}\right)\right\|_{L^{s}}+C\left\|\left(\frac{k(x)}{\mu\left(\mathcal{C}_{h}^{n-1}\right)}-\frac{k(x)}{\mu\left(\mathcal{C}^{n-1}\right)}\right) \nabla P^{n-1}\right\|_{L^{s}} \\
& \quad+C\left\|\overline{\mathbf{P}}_{h} P^{n-1}-P^{n-1}\right\|_{W^{1, s}} \\
& \leq C\left\|\mathcal{C}^{n-1}-\mathcal{C}_{h}^{n-1}\right\|_{L^{\infty}}\left\|P^{n-1}-P_{h}^{n-1}\right\|_{W^{1, s}}+C\left\|\mathcal{C}^{n-1}-\mathcal{C}_{h}^{n-1}\right\|_{L^{s}}\left\|P^{n-1}\right\|_{W^{1, \infty}}+C h\left\|P^{n-1}\right\|_{W^{2, s}} \\
& \leq C h^{\frac{1}{2}}\left\|P^{n-1}-P_{h}^{n-1}\right\|_{W^{1, s}}+C\left\|\mathcal{C}^{n-1}-\mathcal{C}_{h}^{n-1}\right\|_{L^{s}}+C h, \quad n=1, \ldots, m, \quad \forall s \in(1, \infty),
\end{aligned}
$$

where we have used the induction assumption (5.2) to estimate $\left\|\mathcal{C}^{n-1}-\mathcal{C}_{h}^{n-1}\right\|_{L^{\infty}}$, and Lemma 4.1 to estimate $\left\|P^{n-1}\right\|_{W^{1, \infty}}$ and $\left\|P^{n-1}\right\|_{W^{2, s}}$. Choosing $s=4 d$ in the last equation, we can see that when $h \leq h_{2}$ for some $h_{2}>0$,

$$
\left\|P^{n-1}-P_{h}^{n-1}\right\|_{W^{1,4 d}} \leq C\left\|\mathcal{C}^{n-1}-\mathcal{C}_{h}^{n-1}\right\|_{L^{4 d}}+C h, \quad n=1, \ldots, m .
$$

By an inverse inequality,

$$
\begin{aligned}
& \left\|P^{n-1}-P_{h}^{n-1}\right\|_{W^{1, \infty}} \leq\left\|P^{n-1}-\overline{\mathbf{P}}_{h} P^{n-1}\right\|_{W^{1, \infty}}+\left\|\overline{\mathbf{P}}_{h} P^{n-1}-P_{h}^{n-1}\right\|_{W^{1, \infty}} \\
& \leq C h\left\|P^{n-1}\right\|_{W^{2, \infty}}+C h^{-\frac{1}{4}}\left\|\overline{\mathbf{P}}_{h} P^{n-1}-P_{h}^{n-1}\right\|_{W^{1,4 d}} \\
& \leq C h\left\|P^{n-1}\right\|_{W^{2, \infty}}+C h^{-\frac{1}{4}}\left(\left\|\mathcal{C}^{n-1}-\mathcal{C}_{h}^{n-1}\right\|_{L^{4 d}}+h\right) \quad \text { (use (5.6) here) } \\
& \leq C h+C h^{-\frac{1}{4}}\left(h^{\frac{1}{2}}+h\right) \\
& \leq C h^{\frac{1}{4}}, \quad n=1, \ldots, m, \\
& \text { (use (5.2) here) }
\end{aligned}
$$


where we have used Lemma 4.1 and the induction assumption (5.2). Moreover, subtracting (4.5) from (2.3) and using Lemma 3.1 and Lemma 4.1, we derive

$$
\begin{aligned}
& \left\|\mathbf{U}^{n-1}-\mathbf{U}_{h}^{n-1}\right\|_{L^{s}} \\
& \leq\left\|\frac{k(x)}{\mu\left(\mathcal{C}_{h}^{n-1}\right)} \nabla\left(P_{h}^{n-1}-P^{n-1}\right)+\left(\frac{k(x)}{\mu\left(\mathcal{C}_{h}^{n-1}\right)}-\frac{k(x)}{\mu\left(\mathcal{C}^{n-1}\right)}\right) \nabla P^{n-1}\right\|_{L^{s}} \\
& \leq C\left\|P^{n-1}-P_{h}^{n-1}\right\|_{W^{1, s}}+C\left\|\mathcal{C}^{n-1}-\mathcal{C}_{h}^{n-1}\right\|_{L^{s}}\left\|P^{n-1}\right\|_{W^{1, \infty}} \\
& \leq C\left\|P^{n-1}-P_{h}^{n-1}\right\|_{W^{1, s}}+C\left\|\mathcal{C}^{n-1}-\mathcal{C}_{h}^{n-1}\right\|_{L^{s}}, \quad n=1, \ldots, m, \quad \forall s \in[1, \infty] .
\end{aligned}
$$

Setting $s=\infty$ in the inequality above and using (5.7) and the induction assumption (5.2), we obtain

$$
\begin{aligned}
& \left\|\mathbf{U}^{n-1}-\mathbf{U}_{h}^{n-1}\right\|_{L^{\infty}} \\
& \leq C\left\|P^{n-1}-P_{h}^{n-1}\right\|_{W^{1, \infty}}+C\left\|\mathcal{C}^{n-1}-\mathcal{C}_{h}^{n-1}\right\|_{L^{\infty}} \leq C h^{\frac{1}{4}}, \quad n=1, \ldots, m .
\end{aligned}
$$

Similarly, choosing $s=q$ in (5.5) and (5.8), we have

$$
\begin{aligned}
& \left\|\mathbf{U}^{n-1}-\mathbf{U}_{h}^{n-1}\right\|_{L^{q}}+\left\|P^{n-1}-P_{h}^{n-1}\right\|_{W^{1, q}} \\
& \leq C\left\|\mathcal{C}^{n-1}-\mathcal{C}_{h}^{n-1}\right\|_{L^{q}}+C h, \quad n=1, \ldots, m .
\end{aligned}
$$

To estimate $\left\|\mathcal{C}^{n-1}-\mathcal{C}_{h}^{n-1}\right\|_{L^{q}}$, we rewrite the finite element system (2.2) as

$$
\begin{aligned}
\left(\gamma D_{\tau} \mathcal{C}_{h}^{n}, w_{h}\right)+\left(D\left(\mathbf{U}^{n-1}\right) \nabla \mathcal{C}_{h}^{n}, \nabla w_{h}\right)+\left(\mathcal{C}_{h}^{n}, w_{h}\right) \\
=\left(\hat{c} q_{I}^{n}+\left(1-\frac{1}{2}\left(q_{I}^{n}+q_{P}^{n}\right)\right) \mathcal{C}^{n}, w_{h}\right)-\frac{1}{2}\left(\mathbf{U}^{n-1} \cdot \nabla \mathcal{C}^{n}, w_{h}\right)+\frac{1}{2}\left(\mathbf{U}^{n-1} \cdot \nabla w_{h}, \mathcal{C}^{n}\right) \\
\quad+\left(\left(D\left(\mathbf{U}^{n-1}\right)-D\left(\mathbf{U}_{h}^{n-1}\right)\right) \nabla \mathcal{C}_{h}^{n}, \nabla w_{h}\right)+\left(\left(1-\frac{1}{2}\left(q_{I}^{n}+q_{P}^{n}\right)\right)\left(\mathcal{C}_{h}^{n}-\mathcal{C}^{n}\right), w_{h}\right) \\
\quad-\frac{1}{2}\left(\left(\mathbf{U}_{h}^{n-1}-\mathbf{U}^{n-1}\right) \cdot \nabla \mathcal{C}_{h}^{n}, w_{h}\right)+\frac{1}{2}\left(\left(q_{I}^{n-1}-q_{P}^{n-1}\right)\left(\mathcal{C}_{h}^{n}-\mathcal{C}^{n}\right), w_{h}\right) \\
\quad+\frac{1}{2}\left(\left(\mathbf{U}_{h}^{n-1}-\mathbf{U}^{n-1}\right) \cdot \nabla w_{h}, \mathcal{C}_{h}^{n}\right)+\left(\mathbf{U}^{n-1} \cdot \nabla w_{h}, \mathcal{C}_{h}^{n}-\mathcal{C}^{n}\right), \quad \forall w_{h} \in S_{h}^{1} .
\end{aligned}
$$

In view of the difference between the right-hand sides of (4.2) and (5.11), and in order to invoke Lemma 3.2, we define $\theta^{n}$ to be the solution of the following auxiliary time-discrete equation

$$
\begin{aligned}
\gamma D_{\tau} \theta^{n}-\nabla \cdot\left(D\left(\mathbf{U}^{n-1}\right) \nabla \theta^{n}\right)+\theta^{n} \\
\left.=-\nabla \cdot\left(D\left(\mathbf{U}^{n-1}\right)-D\left(\mathbf{U}_{h}^{n-1}\right)\right) \nabla \mathcal{C}_{h}^{n}\right)+\left(1-\frac{1}{2}\left(q_{I}^{n}+q_{P}^{n}\right)\right)\left(\mathcal{C}_{h}^{n}-\mathcal{C}^{n}\right) \\
\quad-\frac{1}{2}\left(\mathbf{U}_{h}^{n-1}-\mathbf{U}^{n-1}\right) \cdot \nabla \mathcal{C}_{h}^{n}+\frac{1}{2}\left(q_{I}^{n-1}-q_{P}^{n-1}\right)\left(\mathcal{C}_{h}^{n}-\mathcal{C}^{n}\right) \\
\quad-\frac{1}{2} \nabla \cdot\left(\left(\mathbf{U}_{h}^{n-1}-\mathbf{U}^{n-1}\right) \mathcal{C}_{h}^{n}\right)-\nabla \cdot\left(\mathbf{U}^{n-1}\left(\mathcal{C}_{h}^{n}-\mathcal{C}^{n}\right)\right),
\end{aligned}
$$

with the boundary and initial conditions

$$
\begin{aligned}
& -D\left(\mathbf{U}^{n-1}\right) \nabla \theta^{n} \cdot \mathbf{n}=-\left(D\left(\mathbf{U}^{n-1}\right)-D\left(\mathbf{U}_{h}^{n-1}\right)\right) \nabla \mathcal{C}_{h}^{n} \cdot \mathbf{n}-\frac{1}{2}\left(\mathbf{U}_{h}^{n-1}-\mathbf{U}^{n-1}\right) \mathcal{C}_{h}^{n} \cdot \mathbf{n} \\
& -\mathbf{U}^{n-1}\left(\mathcal{C}_{h}^{n}-\mathcal{C}^{n}\right) \cdot \mathbf{n} \\
& \theta^{0}=0 \\
& \text { in } \Omega \text {, }
\end{aligned}
$$

and define $\theta_{h}^{n} \in S_{h}^{1}$ to be the solution of the corresponding fully-discrete finite element system:

$$
\begin{aligned}
& \left(\gamma D_{\tau} \theta_{h}^{n}, w_{h}\right)+\left(\left(D\left(\mathbf{U}^{n-1}\right) \nabla \theta_{h}^{n}, \nabla w_{h}\right)+\left(\theta_{h}^{n}, w_{h}\right)\right. \\
& =\left(\left(D\left(\mathbf{U}^{n-1}\right)-D\left(\mathbf{U}_{h}^{n-1}\right)\right) \nabla \mathcal{C}_{h}^{n}, \nabla w_{h}\right)+\left(\left(1-\frac{1}{2}\left(q_{I}^{n}+q_{P}^{n}\right)\right)\left(\mathcal{C}_{h}^{n}-\mathcal{C}^{n}\right), w_{h}\right) \\
& \quad-\frac{1}{2}\left(\left(\mathbf{U}_{h}^{n-1}-\mathbf{U}^{n-1}\right) \cdot \nabla \mathcal{C}_{h}^{n}, w_{h}\right)+\frac{1}{2}\left(\left(q_{I}^{n-1}-q_{P}^{n-1}\right)\left(\mathcal{C}_{h}^{n}-\mathcal{C}^{n}\right), w_{h}\right) \\
& \quad+\frac{1}{2}\left(\left(\mathbf{U}_{h}^{n-1}-\mathbf{U}^{n-1}\right) \cdot \nabla w_{h}, \mathcal{C}_{h}^{n}\right)+\left(\mathbf{U}^{n-1} \cdot \nabla w_{h}, \mathcal{C}_{h}^{n}-\mathcal{C}^{n}\right), \quad \forall w_{h} \in S_{h}^{1},
\end{aligned}
$$

with the initial condition $\theta_{h}^{0}=0$. From (5.12) and (5.13) we see that $\theta_{h}^{n}-\theta^{n}$ satisfies the equation

$$
\begin{array}{r}
\left(\gamma D_{\tau}\left(\theta_{h}^{n}-\theta^{n}\right), w_{h}\right)+\left(\left(D\left(\mathbf{U}^{n-1}\right) \nabla\left(\theta_{h}^{n}-\theta^{n}\right), \nabla w_{h}\right)+\left(\theta_{h}^{n}-\theta^{n}, w_{h}\right)=0,\right. \\
\forall w_{h} \in S_{h}^{1} .
\end{array}
$$


Similarly, subtracting (5.13) and (4.2) from (5.11) gives

$$
\begin{array}{r}
\left(\gamma D_{\tau}\left(\mathcal{C}_{h}^{n}-\theta_{h}^{n}-\mathcal{C}^{n}\right), w_{h}\right)+\left(D\left(\mathbf{U}^{n-1}\right) \nabla\left(\mathcal{C}_{h}^{n}-\theta_{h}^{n}-\mathcal{C}^{n}\right), \nabla w_{h}\right)+\left(\mathcal{C}_{h}^{n}-\theta_{h}^{n}-\mathcal{C}^{n}, w_{h}\right)=0 \\
\forall w_{h} \in S_{h}^{1} .
\end{array}
$$

Here $\mathcal{C}_{h}^{n}-\theta_{h}^{n}$ and $\theta_{h}^{n}$ can be viewed as finite element approximations of $\mathcal{C}^{n}$ and $\theta^{n}$, respectively. In view of (4.7), $D\left(\mathbf{U}^{n-1}\right)$ can be viewed as the value of a piecewise linear function (in time) at time $t_{n-1}$ and therefore, the conditions (3.7)-(3.8) are satisfied. Applying Lemma 3.2 to (5.15) and (5.14) yields

$$
\begin{aligned}
& \left\|D_{\tau}\left(\mathcal{C}_{h}^{n}-\theta_{h}^{n}-\mathbf{P}_{h} \mathcal{C}^{n}\right)\right\|_{L^{p}\left(\widetilde{W}^{-1, q}\right)}+\left\|\mathcal{C}_{h}^{n}-\theta_{h}^{n}-\mathbf{P}_{h} \mathcal{C}^{n}\right\|_{L^{p}\left(W^{1, q}\right)} \\
& \leq C\left(\left\|\mathcal{C}^{n}-\mathbf{R}_{h} \mathcal{C}^{n}\right\|_{L^{p}\left(W^{1, q}\right)}+h^{-1}\left\|\mathbf{P}_{h} \mathcal{C}^{0}-\mathcal{C}_{h}^{0}\right\|_{L^{q}}\right) \\
& \leq C h\left\|\mathcal{C}^{n}\right\|_{L^{p}\left(W^{2, q}\right)}+C h\left\|\mathcal{C}^{0}\right\|_{W^{2, q}}, \quad n=1, \ldots, m . \quad \text { (use (3.1), (3.2) and (3.4)) }
\end{aligned}
$$

and

$$
\begin{aligned}
\left\|D_{\tau}\left(\theta_{h}^{n}-\theta^{n}\right)\right\|_{L^{p}\left(\widetilde{W}^{-1, q}\right)}+\left\|\theta_{h}^{n}-\theta^{n}\right\|_{L^{p}\left(W^{1, q}\right)} \\
\leq C\left\|D_{\tau}\left(\theta_{h}^{n}-\mathbf{P}_{h} \theta^{n}\right)\right\|_{L^{p}\left(\widetilde{W}^{-1, q}\right)}+C\left\|\theta_{h}^{n}-\mathbf{P}_{h} \theta^{n}\right\|_{L^{p}\left(W^{1, q}\right)} \\
\quad+C\left\|D_{\tau} \theta^{n}-\mathbf{P}_{h} D_{\tau} \theta^{n}\right\|_{L^{p}\left(\widetilde{W}^{-1, q}\right)}+C\left\|\theta^{n}-\mathbf{P}_{h} \theta^{n}\right\|_{L^{p}\left(W^{1, q}\right)} \\
\leq C\left\|\theta^{n}-\mathbf{R}_{h} \theta^{n}\right\|_{L^{p}\left(W^{1, q}\right)}+C\left\|D_{\tau^{n}}\right\|_{L^{p}\left(\widetilde{W}^{-1, q}\right)}+C\left\|\theta^{n}\right\|_{L^{p}\left(W^{1, q}\right)} \\
\leq C\left\|D_{\tau} \theta^{n}\right\|_{L^{P}\left(\widetilde{W}^{-1, q}\right)}+C\left\|\theta^{n}\right\|_{L^{p}\left(W^{1, q}\right)}, \quad n=1, \ldots, m,
\end{aligned}
$$

where we have used (3.2) to derive the last inequality, and (3.1) to get the second last inequality (with $m=\ell_{0}=1$ and the dual case $m=\ell_{0}=-1$ ). Therefore,

$$
\left\|D_{\tau} \theta_{h}^{n}\right\|_{L^{p}\left(\widetilde{W}^{-1, q)}\right.}+\left\|\theta_{h}^{n}\right\|_{L^{p}\left(W^{1, q}\right)} \leq C\left(\left\|D_{\tau} \theta^{n}\right\|_{L^{p}\left(\widetilde{W}^{-1, q}\right)}+\left\|\theta^{n}\right\|_{L^{p}\left(W^{1, q}\right)}\right) .
$$

Applying Lemma 3.1 to (5.12) leads to

$$
\begin{aligned}
& \left\|D_{\tau} \theta^{n}\right\|_{L^{p}\left(\widetilde{W}^{-1, q}\right)}+\left\|\theta^{n}\right\|_{L^{p}\left(W^{1, q}\right)} \\
& \leq C\left\|\left(D\left(\mathbf{U}_{h}^{n-1}\right)-D\left(\mathbf{U}^{n-1}\right)\right) \nabla \mathcal{C}_{h}^{n}\right\|_{L^{p}\left(L^{q}\right)}+C\left\|\left(1-\frac{1}{2}\left(q_{I}^{n}+q_{P}^{n}\right)\right)\left(\mathcal{C}_{h}^{n}-\mathcal{C}^{n}\right)\right\|_{L^{p}\left(L^{q}\right)} \\
& \quad+C\left\|\left(\mathbf{U}_{h}^{n-1}-\mathbf{U}^{n-1}\right) \cdot \nabla \mathcal{C}_{h}^{n}\right\|_{L^{p}\left(L^{q}\right)}+C\left\|\left(q_{I}^{n-1}-q_{P}^{n-1}\right)\left(\mathcal{C}_{h}^{n}-\mathcal{C}^{n}\right)\right\|_{L^{p}\left(L^{q}\right)} \\
& \quad+C\left\|\left(\mathbf{U}_{h}^{n-1}-\mathbf{U}^{n-1}\right) \mathcal{C}_{h}^{n}\right\|_{L^{p}\left(L^{q}\right)}+C\left\|\mathbf{U}^{n-1}\left(\mathcal{C}_{h}^{n}-\mathcal{C}^{n}\right)\right\|_{L^{p}\left(L^{q}\right)} \\
& = \\
& =I_{1}^{n}+I_{2}^{n}+I_{3}^{n}+I_{4}^{n}+I_{5}^{n}+I_{6}^{n} .
\end{aligned}
$$

By (5.9)-(5.10), we have the estimate

$$
\begin{aligned}
I_{1}^{n} & =C\left\|\left(D\left(\mathbf{U}_{h}^{n-1}\right)-D\left(\mathbf{U}^{n-1}\right)\right) \nabla \mathcal{C}_{h}^{n}\right\|_{L^{p}\left(L^{q}\right)} \\
& \leq C\left\|\left(D\left(\mathbf{U}_{h}^{n-1}\right)-D\left(\mathbf{U}^{n-1}\right)\right) \nabla\left(\mathcal{C}_{h}^{n}-\mathcal{C}^{n}\right)\right\|_{L^{p}\left(L^{q}\right)}+C\left\|\left(D\left(\mathbf{U}_{h}^{n-1}\right)-D\left(\mathbf{U}^{n-1}\right)\right) \nabla \mathcal{C}^{n}\right\|_{L^{p}\left(L^{q}\right)} \\
& \leq C\left\|\mathbf{U}_{h}^{n-1}-\mathbf{U}^{n-1}\right\|_{L^{\infty}\left(L^{\infty}\right)}\left\|\nabla\left(\mathcal{C}_{h}^{n}-\mathcal{C}^{n}\right)\right\|_{L^{p}\left(L^{q}\right)}+C\left\|\mathbf{U}_{h}^{n-1}-\mathbf{U}^{n-1}\right\|_{L^{p}\left(L^{q}\right)}\left\|\nabla \mathcal{C}^{n}\right\|_{L^{\infty}\left(L^{\infty}\right)} \\
& \leq C h^{\frac{1}{4}}\left\|\nabla\left(\mathcal{C}_{h}^{n}-\mathcal{C}^{n}\right)\right\|_{L^{p}\left(L^{q}\right)}+C\left(\left\|\mathcal{C}_{h}^{n-1}-\mathcal{C}^{n-1}\right\|_{L^{p}\left(L^{q}\right)}+h\right) . \quad n=1, \ldots, m,
\end{aligned}
$$

Similarly, we get

$$
\begin{aligned}
I_{3}^{n} & =C\left\|\left(\mathbf{U}_{h}^{n-1}-\mathbf{U}^{n-1}\right) \nabla \mathcal{C}_{h}^{n}\right\|_{L^{p}\left(L^{q}\right)} \\
& \leq C h^{\frac{1}{4}}\left\|\nabla\left(\mathcal{C}_{h}^{n}-\mathcal{C}^{n}\right)\right\|_{L^{p}\left(L^{q}\right)}+C\left(\left\|\mathcal{C}_{h}^{n-1}-\mathcal{C}^{n-1}\right\|_{L^{p}\left(L^{q}\right)}+h\right), \\
& I_{5}^{n}=C\left\|\left(\mathbf{U}_{h}^{n-1}-\mathbf{U}^{n-1}\right) \mathcal{C}_{h}^{n}\right\|_{L^{p}\left(L^{q}\right)} \leq C h^{\frac{1}{4}}\left\|\mathcal{C}_{h}^{n}-\mathcal{C}^{n}\right\|_{L^{p}\left(L^{q}\right)}+C\left(\left\|\mathcal{C}_{h}^{n-1}-\mathcal{C}^{n-1}\right\|_{L^{p}\left(L^{q}\right)}+h\right),
\end{aligned}
$$

and also

$$
I_{2}^{n}+I_{4}^{n}+I_{6}^{n} \leq C\left\|\mathcal{C}_{h}^{n}-\mathcal{C}^{n}\right\|_{L^{p}\left(L^{q}\right)} .
$$

Substituting the estimates of $I_{j}^{n}, j=1, \ldots, 6$, into (5.18)-(5.19), we obtain

$$
\left\|D_{\tau} \theta_{h}^{n}\right\|_{L^{p}\left(\widetilde{W}^{-1, q}\right)}+\left\|\theta_{h}^{n}\right\|_{L^{p}\left(W^{1, q}\right)}
$$




$$
\leq C h^{\frac{1}{4}}\left\|\nabla\left(\mathcal{C}_{h}^{n}-\mathcal{C}^{n}\right)\right\|_{L^{p}\left(L^{q}\right)}+C\left\|\mathcal{C}_{h}^{n}-\mathcal{C}^{n}\right\|_{L^{p}\left(L^{q}\right)}+C h, \quad n=1, \ldots, m,
$$

which together with (5.16) implies

$$
\begin{aligned}
& \left\|D_{\tau}\left(\mathcal{C}_{h}^{n}-\mathbf{P}_{h} \mathcal{C}^{n}\right)\right\|_{L^{p}\left(\widetilde{W}^{-1, q}\right)}+\left\|\mathcal{C}_{h}^{n}-\mathbf{P}_{h} \mathcal{C}^{n}\right\|_{L^{p}\left(W^{1, q}\right)} \\
& \leq\left\|D_{\tau}\left(\mathcal{C}_{h}^{n}-\theta_{h}^{n}-\mathbf{P}_{h} \mathcal{C}^{n}\right)\right\|_{L^{p}\left(\widetilde{W}^{-1, q}\right)}+\left\|\mathcal{C}_{h}^{n}-\theta_{h}^{n}-\mathbf{P}_{h} \mathcal{C}^{n}\right\|_{L^{p}\left(W^{1, q}\right)} \\
& \quad+\left\|D_{\tau} \theta_{h}^{n}\right\|_{L^{p}(\widetilde{W}-1, q)}+\left\|\theta_{h}^{n}\right\|_{L^{p}\left(W^{1, q}\right)} \\
& \leq C h^{\frac{1}{4}}\left\|\nabla\left(\mathcal{C}_{h}^{n}-\mathcal{C}^{n}\right)\right\|_{L^{p}\left(L^{q}\right)}+C\left\|\mathcal{C}_{h}^{n}-\mathcal{C}^{n}\right\|_{L^{p}\left(L^{q}\right)}+C h \\
& \leq C h^{\frac{1}{4}}\left\|\nabla\left(\mathcal{C}_{h}^{n}-\mathbf{P}_{h} \mathcal{C}^{n}\right)\right\|_{L^{p}\left(L^{q}\right)}+C\left\|\mathcal{C}_{h}^{n}-\mathbf{P}_{h} \mathcal{C}^{n}\right\|_{L^{p}\left(L^{q}\right)}+C h, \quad n=1, \ldots, m,
\end{aligned}
$$

where we have used (3.1) to derive the last inequality. When $h \leq h_{3}$ for some $h_{3}>0$, we can get from above result that

$$
\left\|D_{\tau}\left(\mathcal{C}_{h}^{n}-\mathbf{P}_{h} \mathcal{C}^{n}\right)\right\|_{L^{p}\left(\widetilde{W}^{-1, q)}\right.}+\left\|\mathcal{C}_{h}^{n}-\mathbf{P}_{h} \mathcal{C}^{n}\right\|_{L^{p}\left(W^{1, q}\right)} \leq C\left\|\mathcal{C}_{h}^{n}-\mathbf{P}_{h} \mathcal{C}^{n}\right\|_{L^{p}\left(L^{q}\right)}+C h .
$$

By using (3.4) and the triangle inequality, we further derive that

$$
\begin{array}{r}
\left\|D_{\tau}\left(\mathcal{C}_{h}^{n}-\Pi_{h} \mathcal{C}^{n}\right)\right\|_{L^{p}\left(\widetilde{W}^{-1, q}\right)}+\left\|\mathcal{C}_{h}^{n}-\Pi_{h} \mathcal{C}^{n}\right\|_{L^{p}\left(W^{1, q}\right)} \leq C\left\|\mathcal{C}_{h}^{n}-\Pi_{h} \mathcal{C}^{n}\right\|_{L^{p}\left(L^{q}\right)}+C h \\
n=1, \ldots, m
\end{array}
$$

and by Lemma 3.6,

$$
\begin{aligned}
\left\|\mathcal{C}_{h}^{n}-\Pi_{h} \mathcal{C}^{n}\right\|_{L^{\infty}\left(L^{\infty}\right)} & \leq C\left(\left\|D_{\tau}\left(\mathcal{C}_{h}^{n}-\Pi_{h} \mathcal{C}^{n}\right)\right\|_{L^{p}\left(\widetilde{W}^{-1, q}\right)}+\left\|\mathcal{C}_{h}^{n}-\Pi_{h} \mathcal{C}^{n}\right\|_{L^{p}\left(W^{1, q}\right)}\right) \\
& \leq C\left\|\mathcal{C}_{h}^{n}-\Pi_{h} \mathcal{C}^{n}\right\|_{L^{p}\left(L^{q}\right)}+C h \\
& \leq \frac{1}{2}\left\|\mathcal{C}_{h}^{n}-\Pi_{h} \mathcal{C}^{n}\right\|_{L^{\infty}\left(L^{\infty}\right)}+C\left\|\mathcal{C}_{h}^{n}-\Pi_{h} \mathcal{C}^{n}\right\|_{L^{1}\left(L^{\infty}\right)}+C h, \quad n=1, \ldots, m,
\end{aligned}
$$

Applying Gronwall's inequality, we see that

$$
\left\|\mathcal{C}_{h}^{n}-\Pi_{h} \mathcal{C}^{n}\right\|_{L^{\infty}\left(L^{\infty}\right)} \leq C h, \quad n=1, \ldots, m
$$

Finally, using (3.1), (3.4) and the triangle inequality, we have

$$
\begin{aligned}
\left\|\mathcal{C}_{h}^{n}-\mathbf{P}_{h} \mathcal{C}^{n}\right\|_{L^{\infty}\left(L^{\infty}\right)} & \leq\left\|\mathcal{C}_{h}^{n}-\Pi_{h} \mathcal{C}^{n}\right\|_{L^{\infty}\left(L^{\infty}\right)}+\left\|\Pi_{h} \mathcal{C}^{n}-\mathbf{P}_{h} \mathcal{C}^{n}\right\|_{L^{\infty}\left(L^{\infty}\right)} \\
& \leq C h+C h\left\|\mathcal{C}^{n}\right\|_{L^{\infty}\left(W^{1, \infty}\right)} \leq C h, \quad n=1, \ldots, m,
\end{aligned}
$$

which completes the mathematical induction on (5.2) when $h \leq h_{3}$ for some $h_{3}>0$. Consequently, (5.26) holds for $m=N$ and (5.9) holds for $m=N+1$.

By an inverse inequality and (5.26), we have

$$
\left\|\mathbf{P}_{h} \mathcal{C}^{n}-\mathcal{C}_{h}^{n}\right\|_{L^{\infty}\left(W^{1, \infty}\right)} \leq C h^{-1}\left\|\mathbf{P}_{h} \mathcal{C}^{n}-\mathcal{C}_{h}^{n}\right\|_{L^{\infty}\left(L^{\infty}\right)} \leq C, \quad n=1, \ldots, N
$$

and therefore,

$$
\begin{array}{ll}
\left\|\mathbf{U}_{h}^{n}\right\|_{L^{\infty}} \leq\left\|\mathbf{U}_{h}^{n}-\mathbf{U}^{n}\right\|_{L^{\infty}}+\left\|\mathbf{U}^{n}\right\|_{L^{\infty}} \leq C h^{\frac{1}{4}}+C \leq C, & n=1, \ldots, N, \\
\left\|\mathcal{C}_{h}^{n}\right\|_{W^{1, \infty}} \leq\left\|\mathbf{P}_{h} \mathcal{C}^{n}-\mathcal{C}_{h}^{n}\right\|_{W^{1, \infty}}+\left\|\mathbf{P}_{h} \mathcal{C}^{n}\right\|_{W^{1, \infty}} \leq C+\left\|\mathcal{C}^{n}\right\|_{W^{1, \infty}} \leq C, & n=1, \ldots, N,
\end{array}
$$

where we have used (5.9) to estimate $\left\|\mathbf{U}_{h}^{n}-\mathbf{U}^{n}\right\|_{L^{\infty}}$ and (4.7) for $\left\|\mathbf{U}^{n}\right\|_{L^{\infty}}$ and $\left\|\mathcal{C}^{n}\right\|_{W^{1, \infty}}$, respectively.

The proof of Lemma 5.1 is completed.

\subsection{Proof of (2.5)}

Now we turn back to the proof of Theorem 2.1. We rewrite the system (1.1)-(1.2) into

$$
\begin{aligned}
& -\nabla \cdot\left(\frac{k(x)}{\mu\left(c^{n-1}\right)} \nabla p^{n-1}\right)=q_{I}^{n-1}-q_{P}^{n-1}, \\
& \gamma \partial_{t} c^{n}-\nabla \cdot\left(D\left(\mathbf{u}^{n-1}\right) \nabla c^{n}\right)+c^{n}=\hat{c} q_{I}^{n}+\left(1-\frac{1}{2}\left(q_{I}^{n}+q_{P}^{n}\right)\right) c^{n} \\
& \quad-\frac{1}{2} \mathbf{u}^{n-1} \cdot \nabla c^{n}-\frac{1}{2} \nabla \cdot\left(\mathbf{u}^{n-1} c^{n}\right)+E^{n},
\end{aligned}
$$


where

$$
\mathbf{u}^{n-1}=\frac{k(x)}{\mu\left(c^{n-1}\right)} \nabla p^{n-1},
$$

and $E^{n}$ denotes the truncation error of the linearized scheme, given by

$$
E^{n}=\nabla \cdot\left(\left(D\left(\mathbf{u}^{n}\right)-D\left(\mathbf{u}^{n-1}\right)\right) \nabla c^{n}\right)+\left(\mathbf{u}^{n-1}-\mathbf{u}^{n}\right) \cdot \nabla c^{n}-\frac{1}{2}\left(\left(q_{I}^{n}-q_{P}^{n}\right)-\left(q_{I}^{n-1}-q_{P}^{n-1}\right)\right) c^{n} .
$$

The regularity assumption (2.4) implies

$$
\left\|E^{n}\right\|_{L^{p}\left(L^{q}\right)} \leq C \tau
$$

We subtract (5.28) from (2.1) to get

$$
\begin{aligned}
& \left(\frac{k(x)}{\mu\left(\mathcal{C}_{h}^{n}\right)} \nabla\left(P_{h}^{n}-\overline{\mathbf{P}}_{h} p^{n}\right), \nabla v_{h}\right) \\
& =\left(\frac{k(x)}{\mu\left(\mathcal{C}_{h}^{n}\right)} \nabla\left(p^{n}-\overline{\mathbf{P}}_{h} p^{n}\right), \nabla v_{h}\right)+\left(\left(\frac{k(x)}{\mu\left(c^{n}\right)}-\frac{k(x)}{\mu\left(\mathcal{C}_{h}^{n}\right)}\right) \nabla p^{n}, \nabla v_{h}\right), \quad \forall v_{h} \in \dot{S}_{h}^{2} .
\end{aligned}
$$

By Lemma 5.1 and Lemma 3.5 ,

$$
\begin{aligned}
\left\|P_{h}^{n}-\overline{\mathbf{P}}_{h} p^{n}\right\|_{W^{1, q}} & \leq C\left\|\frac{k(x)}{\mu\left(\mathcal{C}_{h}^{n}\right)} \nabla\left(p^{n}-\overline{\mathbf{P}}_{h} p^{n}\right)\right\|_{L^{q}}+C\left\|\left(\frac{k(x)}{\mu\left(c^{n}\right)}-\frac{k(x)}{\mu\left(\mathcal{C}_{h}^{n}\right)}\right) \nabla p^{n}\right\|_{L^{q}} \\
& \leq C\left\|p^{n}-\overline{\mathbf{P}}_{h} p^{n}\right\|_{W^{1, q}}+C\left\|c^{n}-\mathcal{C}_{h}^{n}\right\|_{L^{q}} \\
& \leq C h^{2}\left\|p^{n}\right\|_{W^{3, q}}+C\left\|c^{n}-\mathcal{C}_{h}^{n}\right\|_{L^{q}}, \quad n=0,1, \ldots, N .
\end{aligned}
$$

Moreover, subtracting (5.30) from (2.3) yields

$$
\begin{aligned}
\left\|\mathbf{u}^{n}-\mathbf{U}_{h}^{n}\right\|_{L^{q}} & \leq\left\|\frac{k(x)}{\mu\left(\mathcal{C}_{h}^{n}\right)} \nabla\left(P_{h}^{n}-p^{n}\right)+\left(\frac{k(x)}{\mu\left(\mathcal{C}_{h}^{n}\right)}-\frac{k(x)}{\mu\left(c^{n}\right)}\right) \nabla p^{n}\right\|_{L^{q}} \\
& \leq C\left\|P_{h}^{n}-p^{n}\right\|_{W^{1, q}}+C\left\|\mathcal{C}_{h}^{n}-c^{n}\right\|_{L^{q}}\left\|p^{n}\right\|_{W^{1, \infty}} \\
& \leq C h^{2}\left\|p^{n}\right\|_{W^{3, q}}+C\left\|\mathcal{C}_{h}^{n}-c^{n}\right\|_{L^{q}}, \quad n=0,1, \ldots, N,
\end{aligned}
$$

where we have used (5.31) to derive the last inequality.

We take the same approach as used for $\left\|\mathcal{C}^{n}-\mathcal{C}_{h}^{n}\right\|_{L^{q}}$ in the last subsection to estimate $\left\|c^{n}-\mathcal{C}_{h}^{n}\right\|_{L^{q}}$. We rewrite the finite element system (2.2) into

$$
\begin{aligned}
\left(\gamma D_{\tau} \mathcal{C}_{h}^{n}, w_{h}\right)+\left(D\left(\mathbf{u}^{n-1}\right) \nabla \mathcal{C}_{h}^{n}, \nabla w_{h}\right)+\left(\mathcal{C}_{h}^{n}, w_{h}\right) \\
=\left(\hat{c} q_{I}^{n}+\left(1-\frac{1}{2}\left(q_{I}^{n}+q_{P}^{n}\right)\right) c^{n}, w_{h}\right)-\frac{1}{2}\left(\mathbf{u}^{n-1} \cdot \nabla c^{n}, w_{h}\right)+\frac{1}{2}\left(\mathbf{u}^{n-1} \cdot \nabla w_{h}, c^{n}\right)+\left(E^{n}, w_{h}\right) \\
\quad+\left(\left(D\left(\mathbf{u}^{n-1}\right)-D\left(\mathbf{U}_{h}^{n-1}\right)\right) \nabla \mathcal{C}_{h}^{n}, \nabla w_{h}\right)+\left(\left(1-\frac{1}{2}\left(q_{I}^{n}+q_{P}^{n}\right)\right)\left(\mathcal{C}_{h}^{n}-c^{n}\right), w_{h}\right) \\
\quad-\frac{1}{2}\left(\left(\mathbf{U}_{h}^{n-1}-\mathbf{u}^{n-1}\right) \cdot \nabla \mathcal{C}_{h}^{n}, w_{h}\right)+\frac{1}{2}\left(\left(q_{I}^{n-1}-q_{P}^{n-1}\right)\left(\mathcal{C}_{h}^{n}-c^{n}\right), w_{h}\right) \\
\quad+\frac{1}{2}\left(\left(\mathbf{U}_{h}^{n-1}-\mathbf{u}^{n-1}\right) \cdot \nabla w_{h}, \mathcal{C}_{h}^{n}\right)+\left(\mathbf{u}^{n-1} \cdot \nabla w_{h}, \mathcal{C}_{h}^{n}-c^{n}\right)-\left(E^{n}, w_{h}\right), \quad \forall w_{h} \in S_{h}^{1} .
\end{aligned}
$$

In view of the difference between the right-hand sides of (5.29) and (5.33), and in order to invoke Lemma 3.2, we define $\chi^{n}$ to be the solution of an auxiliary parabolic equation:

$$
\begin{aligned}
\gamma & D_{\tau} \chi^{n}-\nabla \cdot\left(D\left(\mathbf{u}^{n-1}\right) \nabla \chi^{n}\right)+\chi^{n} \\
= & -\nabla \cdot\left(\left(D\left(\mathbf{u}^{n-1}\right)-D\left(\mathbf{U}_{h}^{n-1}\right)\right) \nabla \mathcal{C}_{h}^{n}\right)+\left(1-\frac{1}{2}\left(q_{I}^{n}+q_{P}^{n}\right)\right)\left(\mathcal{C}_{h}^{n}-c^{n}\right) \\
& -\frac{1}{2}\left(\mathbf{U}_{h}^{n-1}-\mathbf{u}^{n-1}\right) \cdot \nabla \mathcal{C}_{h}^{n}+\frac{1}{2}\left(q_{I}^{n-1}-q_{P}^{n-1}\right)\left(\mathcal{C}_{h}^{n}-c^{n}\right) \\
& -\frac{1}{2} \nabla \cdot\left(\left(\mathbf{U}_{h}^{n-1}-\mathbf{u}^{n-1}\right) \mathcal{C}_{h}^{n}\right)-\nabla \cdot\left(\mathbf{u}^{n-1}\left(\mathcal{C}_{h}^{n}-c^{n}\right)\right)-E^{n},
\end{aligned}
$$

with the boundary and initial conditions

$$
\begin{array}{lll}
-D\left(\mathbf{u}^{n-1}\right) \nabla \chi^{n} \cdot \mathbf{n}= & -\left(D\left(\mathbf{u}^{n-1}\right)-D\left(\mathbf{U}_{h}^{n-1}\right)\right) \nabla \mathcal{C}_{h}^{n} \cdot \mathbf{n}-\frac{1}{2}\left(\mathbf{U}_{h}^{n-1}-\mathbf{u}^{n-1}\right) \mathcal{C}_{h}^{n} \cdot \mathbf{n} & \\
& -\mathbf{u}^{n-1}\left(\mathcal{C}_{h}^{n}-c^{n}\right) \cdot \mathbf{n} & \text { on } \partial \Omega, \\
\chi^{0}=0 & \text { in } \Omega .
\end{array}
$$

The corresponding finite element approximation of (5.34) is defined as: find $\chi_{h}^{n} \in S_{h}^{1}$, such that

$$
\left(\gamma D_{\tau} \chi_{h}^{n}, w_{h}\right)+\left(D\left(\mathbf{u}^{n-1}\right) \nabla \chi_{h}^{n}, \nabla w_{h}\right)+\left(\chi_{h}^{n}, w_{h}\right)
$$




$$
\begin{aligned}
= & \left(\left(D\left(\mathbf{u}^{n-1}\right)-D\left(\mathbf{U}_{h}^{n-1}\right)\right) \nabla \mathcal{C}_{h}^{n}, \nabla w_{h}\right)+\left(\left(1-\frac{1}{2}\left(q_{I}^{n}+q_{P}^{n}\right)\right)\left(\mathcal{C}_{h}^{n}-c^{n}\right), w_{h}\right) \\
& -\frac{1}{2}\left(\left(\mathbf{U}_{h}^{n-1}-\mathbf{u}^{n-1}\right) \cdot \nabla \mathcal{C}_{h}^{n}, w_{h}\right)+\frac{1}{2}\left(\left(q_{I}^{n-1}-q_{P}^{n-1}\right)\left(\mathcal{C}_{h}^{n}-c^{n}\right), w_{h}\right) \\
& +\frac{1}{2}\left(\left(\mathbf{U}_{h}^{n-1}-\mathbf{u}^{n-1}\right) \cdot \nabla w_{h}, \mathcal{C}_{h}^{n}\right)+\left(\mathbf{u}^{n-1} \cdot \nabla w_{h}, \mathcal{C}_{h}^{n}-c^{n}\right)-\left(E^{n}, w_{h}\right), \quad \forall w_{h} \in S_{h}^{1},
\end{aligned}
$$

with the initial condition $\chi_{h}^{0}=0$. By comparing (5.34) and (5.35), we see that

$$
\begin{array}{r}
\left(\gamma D_{\tau}\left(\chi_{h}^{n}-\chi^{n}\right), w_{h}\right)+\left(D\left(\mathbf{u}^{n-1}\right) \nabla\left(\chi_{h}^{n}-\chi^{n}\right), \nabla w_{h}\right)+\left(\chi_{h}^{n}-\chi^{n}, w_{h}\right)=0, \\
\forall w_{h} \in S_{h}^{1} .
\end{array}
$$

Subtracting (5.35) and (5.29) from (5.33) yields

$$
\begin{array}{r}
\left(\gamma D_{\tau}\left(\mathcal{C}_{h}^{n}-\chi_{h}^{n}\right)-\partial_{t} c^{n}, w_{h}\right)+\left(D\left(\mathbf{u}^{n-1}\right) \nabla\left(\mathcal{C}_{h}^{n}-\chi_{h}^{n}-c^{n}\right), \nabla w_{h}\right)+\left(\mathcal{C}_{h}^{n}-\chi_{h}^{n}-c^{n}, w_{h}\right)=0, \\
\forall w_{h} \in S_{h}^{1} .
\end{array}
$$

Again $\mathcal{C}_{h}^{n}-\chi_{h}^{n}$ can be viewed as the finite element approximation of $c^{n}$. Then by Lemma 3.2 ,

$$
\begin{aligned}
& \left\|\mathcal{C}_{h}^{n}-\chi_{h}^{n}-\mathbf{P}_{h} c^{n}\right\|_{L^{p}\left(L^{q}\right)} \\
& \leq C\left\|\mathbf{P}_{h} c^{n}-\mathbf{R}_{h} c^{n}\right\|_{L^{p}\left(L^{q}\right)}+C\left\|\mathbf{P}_{h} c_{0}-\mathcal{C}_{h}^{0}\right\|_{L^{q}}+C\left\|\partial_{t t} c^{n}\right\|_{L^{p}\left(\widetilde{W}^{-1, q}\right)} \tau \\
& \left.\leq C\left\|\mathcal{C}_{h}^{0}-c_{0}\right\|_{L^{q}}+C\left(\tau+h^{2}\right) \quad \text { (use (3.1)-(3.4) }\right) .
\end{aligned}
$$

Similarly, applying Lemma 3.2 to (5.36) yields

$$
\begin{array}{rlr}
\left\|\chi_{h}^{n}\right\|_{L^{p}\left(L^{q}\right)} & \leq\left\|\chi_{h}^{n}-\mathbf{P}_{h} \chi^{n}\right\|_{L^{p}\left(L^{q}\right)}+\left\|\mathbf{P}_{h} \chi^{n}\right\|_{L^{p}\left(L^{q}\right)} & \text { (triangle inequality) } \\
& \leq C\left(\left\|\mathbf{P}_{h} \chi^{n}-\mathbf{R}_{h} \chi^{n}\right\|_{L^{p}\left(L^{q}\right)}+C\left\|\partial_{t t} \chi\right\|_{L^{p}\left(\widetilde{W}^{-1, q}\right)} \tau\right)+C\left\|\chi^{n}\right\|_{L^{p}\left(L^{q}\right)} & \text { (use (3.12) ) } \\
& \leq C h\left\|\chi^{n}\right\|_{L^{p}\left(W^{1, q}\right)}+C \tau+C\left\|\chi^{n}\right\|_{L^{p}\left(L^{q}\right)} .
\end{array}
$$

Substituting the last inequality into (5.38), we have

$$
\begin{aligned}
\left\|\mathcal{C}_{h}^{n}-\mathbf{P}_{h} c^{n}\right\|_{L^{p}\left(L^{q}\right)} & \leq C\left\|\mathcal{C}_{h}^{0}-c^{0}\right\|_{L^{q}}+C\left(\tau+h^{2}\right)+C h\left\|\chi^{n}\right\|_{L^{p}\left(W^{1, q}\right)}+C \tau+C\left\|\chi^{n}\right\|_{L^{p}\left(L^{q}\right)} \\
& \leq C\left\|\mathcal{C}_{h}^{0}-c^{0}\right\|_{L^{q}}+C\left(\tau+h^{2}\right)+C h\left\|\chi^{n}\right\|_{L^{p}\left(W^{1, q}\right)}+C\left\|\chi^{n}\right\|_{L^{\infty}\left(L^{\infty}\right)},
\end{aligned}
$$

and therefore,

$$
\begin{aligned}
\left\|\mathcal{C}_{h}^{n}-c^{n}\right\|_{L^{p}\left(L^{q}\right)} & \leq\left\|\mathcal{C}_{h}^{n}-\mathbf{P}_{h} c^{n}\right\|_{L^{p}\left(L^{q}\right)}+\left\|\mathbf{P}_{h} c^{n}-c^{n}\right\|_{L^{p}\left(L^{q}\right)} \\
& \leq C\left\|\mathcal{C}_{h}^{0}-c^{0}\right\|_{L^{q}}+C\left(\tau+h^{2}\right)+C h\left\|\chi^{n}\right\|_{L^{p}\left(W^{1, q}\right)}+C\left\|\chi^{n}\right\|_{L^{\infty}\left(L^{\infty}\right)},
\end{aligned}
$$

where we have used (3.1) to estimate $\left\|\mathbf{P}_{h} c^{n}-c^{n}\right\|_{L^{p}\left(L^{q}\right)}$.

Since $2 / p+d / q<1$, there exists $p_{0} \in(2, p)$ such that $2 / p_{0}+d / q<1$. To estimate $\left\|\chi^{n}\right\|_{L^{\infty}\left(L^{\infty}\right)}$, we apply Lemma 3.6 and Lemma 3.1 to (5.34) to get

$$
\begin{aligned}
\left\|\chi^{n}\right\|_{L^{\infty}\left(L^{\infty}\right) \leq} \leq & C\left(\left\|D_{\tau} \chi^{n}\right\|_{L^{p_{0}}\left(\widetilde{W}^{-1, q}\right)}+\left\|\chi^{n}\right\|_{L^{p_{0}}\left(W^{1, q}\right)}\right) \\
\leq & C\left\|\left(D\left(\mathbf{u}^{n-1}\right)-D\left(\mathbf{U}_{h}^{n-1}\right)\right) \nabla \mathcal{C}_{h}^{n}\right\|_{L^{p_{0}}\left(L^{q}\right)}+C\left\|\left(1-\frac{1}{2}\left(q_{I}^{n}+q_{P}^{n}\right)\right)\left(\mathcal{C}_{h}^{n}-c^{n}\right)\right\|_{L^{p_{0}\left(L^{q}\right)}} \\
& +C\left\|\left(\mathbf{U}_{h}^{n-1}-\mathbf{u}^{n-1}\right) \cdot \nabla \mathcal{C}_{h}^{n}\right\|_{L^{p_{0}}\left(L^{q}\right)}+C\left\|\left(q_{I}^{n-1}-q_{P}^{n-1}\right)\left(\mathcal{C}_{h}^{n}-c^{n}\right)\right\|_{L^{p_{0}\left(L^{q}\right)}} \\
& +C\left\|\left(\mathbf{U}_{h}^{n-1}-\mathbf{u}^{n-1}\right) \mathcal{C}_{h}^{n}\right\|_{L^{p_{0}}\left(L^{q}\right)}+C\left\|\mathbf{u}^{n-1}\left(\mathcal{C}_{h}^{n}-c^{n}\right)\right\|_{L^{p_{0}\left(L^{q}\right)}}+C\left\|E^{n}\right\|_{\left.L^{p_{0}\left(L^{q}\right)}\right)} \\
\leq & C\left(\left\|\mathbf{U}_{h}^{n-1}-\mathbf{u}^{n-1}\right\|_{L^{p_{0}\left(L^{q}\right)}}+\left\|\mathcal{C}_{h}^{n}-c^{n}\right\|_{L^{p_{0}\left(L^{q}\right)}}+\left\|E^{n}\right\|_{L^{p_{0}\left(L^{q}\right)}}\right) \\
\leq & C\left\|\mathcal{C}_{h}^{0}-c^{0}\right\|_{L^{q}}+C\left\|\mathcal{C}_{h}^{n}-c^{n}\right\|_{L^{p_{0}\left(L^{q}\right)}}+C\left(\tau+h^{2}\right),
\end{aligned}
$$

where we have used Lemma 5.1 to estimate $\left\|\nabla \mathcal{C}_{h}^{n}\right\|_{L^{\infty}}$ and $\left\|\mathcal{C}_{h}^{n}\right\|_{L^{\infty}}$, and (5.32) in deriving the last inequality. Similarly, replacing $p_{0}$ by $p$ in the last inequality yields

$$
\left(\left\|D_{\tau} \chi^{n}\right\|_{L^{p}\left(\widetilde{W}^{-1, q}\right)}+\left\|\chi^{n}\right\|_{L^{p}\left(W^{1, q}\right)}\right) \leq C\left\|\mathcal{C}_{h}^{0}-c^{0}\right\|_{L^{q}}+C\left\|\mathcal{C}_{h}^{n}-c^{n}\right\|_{L^{p}\left(L^{q}\right)}+C\left(\tau+h^{2}\right) .
$$

By substituting the last two estimates into (5.40), we obtain

$$
\begin{aligned}
& \left\|\mathcal{C}_{h}^{n}-c^{n}\right\|_{L^{p}\left(L^{q}\right)} \\
& \leq C\left\|\mathcal{C}_{h}^{0}-c^{0}\right\|_{L^{q}}+C\left(\tau+h^{2}\right)+C h\left\|\mathcal{C}_{h}^{n}-c^{n}\right\|_{L^{p}\left(L^{q}\right)}+C\left\|\mathcal{C}_{h}^{n}-c^{n}\right\|_{L^{p_{0}}\left(L^{q}\right)}
\end{aligned}
$$




$$
\leq C\left\|\mathcal{C}_{h}^{0}-c^{0}\right\|_{L^{q}}+C\left(\tau+h^{2}\right)+C h\left\|\mathcal{C}_{h}^{n}-c^{n}\right\|_{L^{p}\left(L^{q}\right)}+\frac{1}{2}\left\|\mathcal{C}_{h}^{n}-c^{n}\right\|_{L^{p}\left(L^{q}\right)}+C\left\|\mathcal{C}_{h}^{n}-c^{n}\right\|_{L^{1}\left(L^{q}\right)} .
$$

When $h \leq h_{4}$ for some $h_{4}>0$, we have

$$
\left\|\mathcal{C}_{h}^{n}-c^{n}\right\|_{L^{p}\left(L^{q}\right)} \leq C\left\|\mathcal{C}_{h}^{0}-c^{0}\right\|_{L^{q}}+C\left\|\mathcal{C}_{h}^{n}-c^{n}\right\|_{L^{1}\left(L^{q}\right)}+C\left(\tau+h^{2}\right) .
$$

or equivalently

$$
\left(\tau \sum_{n=1}^{m}\left\|\mathcal{C}_{h}^{n}-c^{n}\right\|_{L^{q}}^{p}\right)^{\frac{1}{p}} \leq C\left\|\mathcal{C}_{h}^{0}-c^{0}\right\|_{L^{q}}+C \tau \sum_{n=1}^{m}\left\|\mathcal{C}_{h}^{n}-c^{n}\right\|_{L^{q}}+C\left(\tau+h^{2}\right) .
$$

By a similar approach, we can obtain the estimate:

$$
\left(\tau \sum_{n=k+1}^{m}\left\|\mathcal{C}_{h}^{n}-c^{n}\right\|_{L^{q}}^{p}\right)^{\frac{1}{p}} \leq C\left\|\mathcal{C}_{h}^{k}-c^{k}\right\|_{L^{q}}+C \tau \sum_{n=k}^{m}\left\|\mathcal{C}_{h}^{n}-c^{n}\right\|_{L^{q}}+C\left(\tau+h^{2}\right) .
$$

By the generalized Gronwall inequality (Lemma 3.7),

$$
\left\|\mathcal{C}_{h}^{N}-c^{N}\right\|_{L^{p}\left(L^{q}\right)} \leq C\left\|\mathcal{C}_{h}^{0}-c^{0}\right\|_{L^{p}\left(L^{q}\right)}+C\left(\tau+h^{2}\right) \leq C\left(\tau+h^{2}\right) .
$$

Finally combining the estimates (5.31)-(5.32) and (5.44), we obtain the following error estimate when $h \leq h_{p, q}=\min _{1 \leq j \leq 4} h_{j}$ and $\tau \leq \tau_{p, q}=\min _{1 \leq j \leq 5} \tau_{j}$,

$$
\left\|P_{h}^{N}-p^{N}\right\|_{L^{p}\left(W^{1, q}\right)}+\left\|\mathbf{U}_{h}^{N}-\mathbf{u}^{N}\right\|_{L^{p}\left(L^{q}\right)}+\left\|\mathcal{C}_{h}^{N}-c^{N}\right\|_{L^{p}\left(L^{q}\right)} \leq C\left(\tau+h^{2}\right) .
$$

Since $q>d$, the inequality above implies (2.5). This proves Theorem 2.1 in the case $\tau \leq \tau_{p, q}$ and $h \leq h_{p, q}$.

\subsection{The case $\tau \geq \tau_{p, q}$ or $h \geq h_{p, q}$}

For any $\tau$ and $h$, substituting $\left(v_{h}, w_{h}\right)=\left(P_{h}^{n-1}, \mathcal{C}_{h}^{n}\right)$ into (2.1)-(2.2) yields

$$
\begin{aligned}
& \left\|\nabla P_{h}^{n-1}\right\|_{L^{2}}^{2} \leq\left\|q_{I}^{n-1}-q_{P}^{n-1}\right\|_{L^{2}}\left\|P_{h}^{n-1}\right\|_{L^{2}} \leq\left\|q_{I}^{n-1}-q_{P}^{n-1}\right\|_{L^{2}}\left\|\nabla P_{h}^{n-1}\right\|_{L^{2}}, \\
& D_{\tau}\left(\frac{\gamma}{2}\left\|\mathcal{C}_{h}^{n}\right\|_{L^{2}}^{2}\right) \leq \frac{\gamma}{4 \tau}\left\|\mathcal{C}_{h}^{n}\right\|_{L^{2}}^{2}+\frac{\tau}{\gamma}\left\|\hat{c} q_{I}^{n}\right\|_{L^{\infty}}^{2},
\end{aligned}
$$

which further imply

$$
\max _{0 \leq n \leq N}\left(\left\|P_{h}^{n}\right\|_{H^{1}}+\left\|\mathcal{C}_{h}^{n}\right\|_{L^{2}}\right) \leq C .
$$

If $\tau \geq \tau_{p, q}$, (5.22) still holds for $h \leq h_{p, q} \leq h_{3}$, which implies that

$$
\begin{aligned}
& \left\|D_{\tau}\left(\mathcal{C}_{h}^{n}-\mathbf{P}_{h} \mathcal{C}^{n}\right)\right\|_{L^{p}\left(\widetilde{W}^{-1, q}\right)}+\left\|\mathcal{C}_{h}^{n}-\mathbf{P}_{h} \mathcal{C}^{n}\right\|_{L^{p}\left(W^{1, q}\right)} \\
& \leq C\left\|\mathcal{C}_{h}^{n}-\mathbf{P}_{h} \mathcal{C}^{n}\right\|_{L^{p}\left(L^{q}\right)}+C h \\
& \leq \frac{1}{2}\left\|\mathcal{C}_{h}^{n}-\mathbf{P}_{h} \mathcal{C}^{n}\right\|_{L^{p}\left(W^{1, q}\right)}+C\left\|\mathcal{C}_{h}^{n}-\mathbf{P}_{h} \mathcal{C}^{n}\right\|_{L^{p}\left(L^{2}\right)}+C h \quad \text { (use (3.25) here) } \\
& \leq \frac{1}{2}\left\|\mathcal{C}_{h}^{n}-\mathbf{P}_{h} \mathcal{C}^{n}\right\|_{L^{p}\left(W^{1, q}\right)}+C,
\end{aligned}
$$

where the last inequality is due to (5.46). Then we see that

$$
\begin{aligned}
\left\|\mathcal{C}_{h}^{n}-c^{n}\right\|_{L^{p}\left(W^{1, q}\right)} & \leq\left\|\mathcal{C}_{h}^{n}-\mathbf{P}_{h} \mathcal{C}^{n}\right\|_{L^{p}\left(W^{1, q}\right)}+\left\|\mathbf{P}_{h} \mathcal{C}^{n}-c^{n}\right\|_{L^{p}\left(W^{1, q}\right)} \\
& \leq C=C \tau_{p, q}^{-1} \tau_{p, q} \leq C \tau_{p, q}^{-1}\left(\tau+h^{2}\right) .
\end{aligned}
$$

On the other hand, (5.7) and (5.9) imply that for $h \leq h_{p, q} \leq h_{2}$,

$$
\begin{aligned}
& \left\|\mathbf{u}^{n}-\mathbf{U}_{h}^{n}\right\|_{L^{\infty}}+\left\|p^{n}-P_{h}^{n}\right\|_{W^{1, \infty}} \\
& \leq\left\|\mathbf{u}^{n}-\mathbf{U}^{n}\right\|_{L^{\infty}}+\left\|p^{n}-P^{n}\right\|_{W^{1, \infty}}+\left\|\mathbf{U}^{n}-\mathbf{U}_{h}^{n}\right\|_{L^{\infty}}+\left\|P^{n}-P_{h}^{n}\right\|_{W^{1, \infty}} \\
& \leq C=C \tau_{p, q}^{-1} \tau_{p, q} \leq C \tau_{p, q}^{-1}\left(\tau+h^{2}\right) .
\end{aligned}
$$

This proves Theorem 2.1 in the case $\tau \geq \tau_{p, q}$ and $h \leq h_{p, q}$.

If $h \geq h_{p, q}$, by (5.46) and an inverse inequality, we have

$$
\max _{0 \leq n \leq N}\left(\left\|P_{h}^{n}\right\|_{W^{1, q}}+\left\|\mathcal{C}_{h}^{n}\right\|_{L^{q}}\right) \leq C h^{\frac{d}{q}-\frac{d}{2}} \max _{0 \leq n \leq N}\left(\left\|P_{h}^{n}\right\|_{H^{1}}+\left\|\mathcal{C}_{h}^{n}\right\|_{L^{2}}\right)
$$




$$
\leq C h_{p, q}^{\frac{d}{q}-\frac{d}{2}} \max _{0 \leq n \leq N}\left(\left\|P_{h}^{n}\right\|_{H^{1}}+\left\|\mathcal{C}_{h}^{n}\right\|_{L^{2}}\right) \leq C,
$$

and therefore, by noting $\left\|\mathbf{U}_{h}^{n}\right\|_{L^{q}} \leq C\left\|P_{h}^{n}\right\|_{W^{1, q}} \leq C$,

$$
\begin{aligned}
& \max _{0 \leq n \leq N}\left(\left\|p^{n}-P_{h}^{n}\right\|_{W^{1, q}}+\left\|\mathbf{u}^{n}-\mathbf{U}_{h}^{n}\right\|_{L^{q}}+\left\|c^{n}-\mathcal{C}_{h}^{n}\right\|_{L^{q}}\right) \\
& \leq C=C h_{p, q}^{-2} h_{p, q}^{2} \leq C h_{p, q}^{-2}\left(\tau+h^{2}\right) .
\end{aligned}
$$

This proves Theorem 2.1 in the case $h \geq h_{p, q}$.

\section{Proof of Corollary 2.2}

By using an inverse inequality noting [Lemma 5.1, (5.1)], we can derive from (2.2) that

$$
\begin{aligned}
\left\|D_{\tau} \mathcal{C}_{h}^{n}\right\|_{L^{p}\left(L^{q}\right)} \leq & C h^{-1}\left\|D\left(\mathbf{U}_{h}^{n-1}\right) \nabla \mathcal{C}_{h}^{n}\right\|_{L^{p}\left(L^{q}\right)}+C\left\|\left(q_{I}^{n}+q_{P}^{n}\right) \mathcal{C}_{h}^{n}\right\|_{L^{p}\left(L^{q}\right)} \\
& +C\left\|\mathbf{U}_{h}^{n-1} \cdot \nabla \mathcal{C}_{h}^{n}\right\|_{L^{p}\left(L^{q}\right)}+C h^{-1}\left\|\mathbf{U}_{h}^{n-1} \mathcal{C}_{h}^{n}\right\|_{L^{p}\left(L^{q}\right)}+C\left\|\hat{c} q_{I}^{n}\right\|_{L^{p}\left(L^{q}\right)} \\
\leq & C h^{-1}\left\|\mathbf{U}_{h}^{n-1}\right\|_{L^{\infty}\left(L^{\infty}\right)}\left\|\mathcal{C}_{h}^{n}\right\|_{L^{\infty}\left(W^{1, \infty}\right)}+C\left\|\mathcal{C}_{h}^{n}\right\|_{L^{\infty}\left(L^{\infty}\right)} \\
& +C\left\|\mathbf{U}_{h}^{n-1}\right\|_{L^{\infty}\left(L^{\infty}\right)}\left\|\mathcal{C}_{h}^{n}\right\|_{L^{\infty}\left(W^{1, \infty}\right)}+C h^{-1}\left\|\mathbf{U}_{h}^{n-1}\right\|_{L^{\infty}\left(L^{\infty}\right)}\left\|\mathcal{C}_{h}^{n}\right\|_{L^{\infty}\left(L^{\infty}\right)}+C \\
\leq & C h^{-1}
\end{aligned}
$$

which in turn shows $\left\|D_{\tau}\left(\mathcal{C}_{h}^{n}-c^{n}\right)\right\|_{L^{p}\left(L^{q}\right)} \leq C h^{-1}$ and

$$
\left\|D_{\tau}\left(\mathcal{C}_{h}^{n}-c^{n}\right)\right\|_{L^{p}\left(L^{q}\right)} \leq C \tau^{-1}\left\|\mathcal{C}_{h}^{n}-c^{n}\right\|_{L^{p}\left(L^{q}\right)} \leq C \tau^{-1}\left(\tau+h^{2}\right) \leq C \tau^{-1} .
$$

Moreover, by the Sobolev interpolation inequality, we have

$$
\begin{aligned}
\left\|\mathcal{C}_{h}^{N}-c^{N}\right\|_{L^{\infty}\left(L^{q}\right)} & \leq\left\|\mathcal{C}_{h}^{0}-c^{0}\right\|_{L^{q}}+C\left\|\mathcal{C}_{h}^{N}-c^{N}\right\|_{L^{p}\left(L^{q}\right)}^{1-\frac{1}{p}}\left\|D_{\tau}\left(\mathcal{C}_{h}^{N}-c^{N}\right)\right\|_{L^{p}\left(L^{q}\right)}^{\frac{1}{p}} \\
& \leq C h^{2}\left\|c^{0}\right\|_{W^{2, q}}+C\left(\tau+h^{2}\right)^{1-\frac{1}{p}} \min \left(\tau^{-1}, h^{-1}\right)^{\frac{1}{p}} \\
& \leq C h^{2}+C\left(\tau^{1-\frac{2}{p}}+h^{2-\frac{3}{p}}\right),
\end{aligned}
$$

where we have used (2.5) to estimate $\left\|\mathcal{C}_{h}^{N}-c^{N}\right\|_{L^{p}\left(L^{q}\right)}$. Since $p$ can be chosen arbitrarily large, combining the above inequality and (5.31)-(5.32), we obtain (2.6) immediately and the proof of Corollary 2.2 is completed.

\section{Numerical results}

In this section we present numerical results to support our theoretical analysis. All the computations are performed by using FreeFEM++ [15].

We consider the equations

$$
\begin{array}{r}
\frac{\partial c}{\partial t}-\nabla \cdot(D(\mathbf{u}) \nabla c)+\mathbf{u} \cdot c=g, \\
-\nabla \cdot\left(\frac{2}{\mu(c)} \nabla p\right)=f
\end{array}
$$

in the circular domain $\Omega=\left\{(x, y):(x-0.5)^{2}+(y-0.5)^{2}<0.5^{2}\right\}$, with

$$
\mathbf{u}=-\frac{2}{\mu(c)} \nabla p, \quad \mu(c)=1+c, \quad D(\mathbf{u})=1+0.1|\mathbf{u}|,
$$

and an artificially constructed exact solution

$$
p=100(x-t)^{2} e^{-t}, \quad c=0.5+0.2 e^{-t} \cos (x) \sin (y) .
$$

Substituting this exact solution into the equations (7.1)-(7.2) yields the source terms $g, f$ and the boundary conditions

$$
\mathbf{u} \cdot \mathbf{n}=f_{b} \quad \text { and } \quad D(\mathbf{u}) \nabla c \cdot \mathbf{n}=g_{b} \quad \text { on } \partial \Omega
$$


These are the same type of boundary conditions with given nonzero right-hand sides.

A quasi-uniform triangulation is made by FreeFEM++ with $M$ nodes uniformly distributed on the boundary of the circular domain. For simplicity, we denote $h=1 / M$. We solve the system (7.1) - (7.4) by the proposed method on the quasi-uniform mesh up to time $T=1$. The $L^{2}$ and $L^{\infty}$ errors of the numerical solutions at time $t=1$ are presented in Table 7.1 with a small fixed time step size $\tau=2^{-14}$ such that the errors from time discretization can be negligible in observing the convergence rate in the spatial direction. We can see from Table 7.1 that the proposed method provides the accuracy of the optimal order $O\left(1 / M^{2}\right)$ for both $\mathcal{C}_{h}^{n}$ and $\mathbf{U}_{h}^{n}$. On the other hand, we present in Table 7.2 the $L^{2}$ and $L^{\infty}$ errors of the numerical solutions with a small fixed mesh size $h=1 / 256$ to show the convergence rate in the temporal direction. From Table 7.2 , one can observe clearly that the accuracy of the proposed method in time direction is of first order. The numerical results are consistent with the analysis given in this paper.

TABLE 7.1. Errors of numerical solutions in spatial direction $\left(\tau=2^{-14}\right)$

\begin{tabular}{c|cccc}
\hline$h$ & $\left\|c^{N}-\mathcal{C}_{h}^{N}\right\|_{L^{2}}$ & $\left\|\mathbf{u}^{N}-\mathbf{U}_{h}^{N}\right\|_{L^{2}}$ & $\left\|c^{N}-\mathcal{C}_{h}^{N}\right\|_{L^{\infty}}$ & $\left\|\mathbf{u}^{N}-\mathbf{U}_{h}^{N}\right\|_{L^{\infty}}$ \\
\hline $1 / 16$ & $1.3995 \mathrm{E}-04$ & $3.0027 \mathrm{E}-03$ & $5.1714 \mathrm{E}-04$ & $1.7159 \mathrm{E}-02$ \\
$1 / 32$ & $2.8838 \mathrm{E}-05$ & $6.9765 \mathrm{E}-04$ & $1.4176 \mathrm{E}-04$ & $5.2594 \mathrm{E}-03$ \\
$1 / 64$ & $7.1872 \mathrm{E}-06$ & $1.7068 \mathrm{E}-04$ & $3.4551 \mathrm{E}-05$ & $1.2412 \mathrm{E}-03$ \\
\hline order & 2.00 & 2.02 & 2.03 & 2.08 \\
\hline
\end{tabular}

TABLE 7.2. Errors of numerical solutions in time direction $(h=1 / 256)$

\begin{tabular}{c|cccc}
\hline$\tau$ & $\left\|c^{N}-\mathcal{C}_{h}^{N}\right\|_{L^{2}}$ & $\left\|\mathbf{u}^{N}-\mathbf{U}_{h}^{N}\right\|_{L^{2}}$ & $\left\|c^{N}-\mathcal{C}_{h}^{N}\right\|_{L^{\infty}}$ & $\left\|\mathbf{u}^{N}-\mathbf{U}_{h}^{N}\right\|_{L^{\infty}}$ \\
\hline $1 / 32$ & $4.1618 \mathrm{E}-04$ & $6.2041 \mathrm{E}-04$ & $2.3635 \mathrm{E}-03$ & $2.4287 \mathrm{E}-03$ \\
$1 / 64$ & $1.8478 \mathrm{E}-04$ & $2.8533 \mathrm{E}-04$ & $1.1310 \mathrm{E}-03$ & $1.0462 \mathrm{E}-03$ \\
$1 / 128$ & $8.5562 \mathrm{E}-05$ & $1.3755 \mathrm{E}-04$ & $5.3595 \mathrm{E}-04$ & $4.7889 \mathrm{E}-04$ \\
\hline order & 1.06 & 1.06 & 1.07 & 1.12 \\
\hline
\end{tabular}

\section{Conclusion}

In this paper, we have presented an error estimate for the system of PDEs governing miscible displacement in porous media with the Bear-Scheidegger diffusion-dispersion coefficient, which is time-dependent and only "Lipschitz continuous". The analysis utilizes the discrete maximal $L^{p}$-regularity of finite element solutions of parabolic equations, which was established in [28, 31, 32 for parabolic equations with Lipschitz continuous coefficients in smooth domains, for timeindependent coefficients, time-dependent coefficients with semi-discrete finite element method, and time-dependent coefficients with fully discrete finite element method, respectively. In these articles (as well as this paper), the domain is assumed to be partitioned into triangles or tetrahedra which fit the boundary $\partial \Omega$ exactly, with possibly curved triangles or tetrahedra near on the boundary.

In the two-dimensional case, the finite element space can be naturally extended (or restricted) to the curved triangle near the boundary. However, in the three-dimensional case, if the boundary faces of the tetrahedra do not exactly lie on $\partial \Omega$ then the curved tetrahedra near the boundary should be specifically constructed instead of being an natural extension of the tetrahedra as in the two-dimensional case. For example, for a point $x$ on a boundary face of a tetrahedron one can associate a unique point $y=y(x) \in \partial \Omega$ such that

$$
y=x+\mathbf{n}(y) d(x)
$$

where $\mathbf{n}(y)$ is the outward unit normal vector on the point $y \in \partial \Omega$, and $d(x)$ is the signed distance from $x$ to $y$. For $x \in \Omega$ there holds $d(x)>0$, and $x \in \mathbb{R}^{d} \backslash \Omega$ there holds $d(x) \leq 0$. Such a transition between the interpolated surface $\partial \Omega_{h}$ and the exact surface $\partial \Omega$ was introduced as a lift operator in [11, 12]. For a tetrahedron $\mathfrak{T}$ with a triangular face $e \subset \partial \Omega_{h}$, the lift of $e$ onto the smooth boundary 
$\partial \Omega$ is a curved triangle on $\partial \Omega$. The lift of all such triangles on $\partial \Omega_{h}$ form a curved triangulation of $\partial \Omega$. One can define a region

$$
\hat{\mathfrak{T}}=\cup_{x \in e}\{x+\theta \nu(y) d(x): \theta \in[0,1)\} .
$$

Then $\hat{\mathfrak{T}}:=\hat{\tau} \cup \tau$ is a curved tetrahedron which fit the boundary exactly.

Such a triangulation with possibly curved tetrahedra on the boundary exists theoretically, as shown above, but is not convenient for practical computation. In practical computation, people often replace the original domain $\Omega$ by a triangulated polygonal/polyhedral domain $\Omega_{h}$. For example, FreeFEM++ solved PDEs in this way. Therefore, our numerical example in Section 7 actually neglects the quadrature error on the boundary triangles (neglecting the quadrature on $\Omega \backslash \Omega_{h}$ ). This gap between theoretical analysis and practical computation by using FreeFEM ++ can possibly be filled in the future by either of the following two approaches:

(1) Instead of assuming that the triangulation fit the boundary exactly, one can use the discrete maximal $L^{p}$-regularity result established by Kashiwabara and Kemmochi 21, who worked on the triangulated domain $\Omega_{h}$ instead of the original domain $\Omega$. In order to apply such results to miscible displacement in porous media, one needs to first extend the result of [21] to parabolic equations with time-dependent Lipschitz continuous coefficients.

(2) Instead of assuming $\Omega$ to be smooth, one can work on a polygonal/polyhedronal domain directly. However, the discrete maximal $L^{p}$-regularity of parabolic equations was only established for the Dirichlet boundary condition so far, see [33]. In order to apply such results to miscible displacement in porous media, one needs to first extend the result of [33] to the Neumann boundary condition. In this case, the error estimates in Theorem 2.1 can only be proved for some $q$ depending on the interior angles of the corners and edges, instead of all $q \in(d, \infty)$.

\section{References}

[1] R. A. Adams, J.F. Fournier, Sobolev spaces, 2003 Elsevier Ltd, Netherlands.

[2] G. Akrivis and B. Li, Maximum norm analysis of implicit-explicit backward difference formulas for nonlinear parabolic equations, IMA J. Numer. Anal., 38 (2018), pp. 75-101.

[3] G. Akrivis, B. Li, and C. Lubich, Combining maximal regularity and energy estimates for time discretizations of quasilinear parabolic equations, Math. Comp., 86 (2017), pp. 1527-1552.

[4] A. Ashyralyev, S. Piskarev and L. Weis, On well-posedness of difference schemes for abstract parabolic equations in $L_{p}([0, T] ; E)$ spaces, Numer. Funct. Anal. Optim., 23 (2002), pp. 669-693.

[5] P. Auscher and M. Qafsaoui, Observations on $W^{1, p}$ estimates for divergence elliptic equations with VMO coefficients, Bollettino dellUnione Matematica Italiana, Serie 8, Vol. 5-B (2002), pp. 487509.

[6] J. Bear and Y. Bachmat, Introduction to modeling of transport phenomena in porous media, Springer-Verlag, New York, 1990.

[7] S. C. Brenner and L. R. Scott, The mathematical theory of finite element methods, 3rd edition, Springer, New York, 2008.

[8] M. Crouzeix, Contractivity and analyticity in $\ell^{p}$ of some approximation of the heat equation. Numerical Algorithms, 33 (2003), pp. 193-201.

[9] A. Demlow, D. Leykekhman, A. H. Schatz and L. B. Wahlbin, Best approximation property in the $W^{1, p}$ norm for finite element methods on graded meshes, Math. Comput., 81(2012), pp. 743-764.

[10] J. Douglas, Jr., R. E. Ewing, and M. F. Wheeler, The approximation of the pressure by a mixed method in the simulation of miscible displacement, R.A.I.R.O. Analyse Numerique, 17 (1983), pp. 17-33.

[11] G. Dziuk, Finite elements for the Beltrami operator on arbitrary surfaces, in "Partial differential equations and calculus of variations", edited by S. Hildebrandt and R. Leis, pp. 142-155, 1988. Lecture Notes in Mathematics, vol 1357. Springer, Berlin, Heidelberg.

[12] G. Dziuk and C. M. Elliott, Finite element methods for surface PDEs, Acta Numerica, 22 (2013), pp. $289-396$.

[13] R. E. Ewing and M. F. Wheeler, Galerkin methods for miscible displacement problems in porous media, SIAM J. Numer. Anal., 17 (1980), pp. 351-365.

[14] X. Feng, On existence and uniqueness results for a coupled system modeling miscible displacement in porous media, J. Math. Anal. Appl., 194 (1995), pp. 883-910.

[15] Freefem++, version 3.51, http://www.freefem.org/.

[16] M. Geissert, Discrete maximal $L^{p}$ regularity for finite element operators, SIAM J. Numer. Anal., 44 (2006), pp. 677-698.

[17] M. Geissert, Applications of discrete maximal $L^{p}$ regularity for finite element operators, Numer. Math., 108 (2007), pp. 121-149.

[18] P. Grisvard: Elliptic Problems in Nonsmooth Domains, SIAM 2011. 
[19] A. Hansbo, Strong stability and non-smooth data error estimates for discretizations of linear parabolic problems, BIT Numer. Math., 42 (2002), pp. 351-379.

[20] Y. Hou, B. Li and W. Sun, Error analysis of splitting Galerkin methods for heat and sweat transport in textile materials, SIAM J. Numer. Anal., 51 (2013), pp. 88-111.

[21] T. Kashiwabara and T. Kemmochi, Maximum norm error estimates for the finite element approximation of parabolic problems on smooth domains, Preprint, arXiv:1805.01336

[22] T. Kemmochi and N. Saito, Discrete maximal regularity and the finite element method for parabolic equations. Preprint. http://arXiv.org/abs/1602.06864

[23] B. Kovács, B. Li, and C. Lubich, A-stability implies discrete maximal parabolic regularity, SIAM J. Numer. Anal., 54 (2016), pp. 3600-3624.

[24] P. C. Kunstmann, B. Li, and C. Lubich, Runge-Kutta time discretization of nonlinear parabolic equations studied via discrete maximal parabolic regularity, Found. Comput. Math., 2017, DOI: 10.1007/s10208-017-9364-x

[25] P.C. Kunstmann and L. Weis, Maximal $L^{p}$-regularity for parabolic equations, Fourier multiplier theorems and $H^{\infty}$-functional calculus, Lecture notes in mathematics, 1855 (2004), pp. 65-311.

[26] D. Leykekhman, Pointwise localized error estimates for parabolic finite element equations, Numer. Math., 96 (2004), pp. 583-600.

[27] D. Leykekhman and B. Vexler: Discrete maximal parabolic regularity for Galerkin finite element methods, Numer. Math., 135 (2017), pp. 923-952.

[28] B. Li, Maximum-norm stability and maximal $L^{p}$ regularity of FEMs for parabolic equations with Lipschitz continuous coefficients, Numer. Math., 131 (2015), pp. 489-516.

[29] B. Li and W. Sun, Unconditional convergence and optimal error estimates of a Galerkin-mixed FEM for incompressible miscible flow in porous media, SIAM J. Numer. Anal., 51 (2013), pp. 1959-1977.

[30] B. Li and W. Sun, Error analysis of linearized semi-implicit Galerkin finite element methods for nonlinear parabolic equations, Int. J. Numer. Anal. Modeling, 10 (2013), pp. 622-633.

[31] B. Li and W. Sun, Regularity of the diffusion-dispersion tensor and error analysis of FEMs for a porous media flow, SIAM J. Numer. Anal., 53(2015), pp. 1418-1437.

[32] B. Li and W. Sun, Maximal regularity of fully discrete finite element solution of parabolic equations, SIAM J. Numer. Anal., 55 (2017), pp. 521-542.

[33] B. Li and W. Sun, Maximal $L^{p}$ analysis of finite element solutions for parabolic equations with nonsmooth coefficients in convex polyhedra. Math. Comp., 86 (2017), pp. 1071-1102.

[34] G. M. Lieberman, Second Order Parabolic Differential Equations, World Scientific Publishing Co. Pte. Ltd, Copyright 1996, Singapore. Reprinted in 2005.

[35] G. M. Lieberman, Oblique Derivative Problems for Elliptic Equations, World Scientific Publishing Co. Pte. Ltd, Singapore, 2013.

[36] Y. Lin, On maximum norm estimates for Ritz-Volterra projection with applications to some time dependent problems, J. Comput. Math., 15 (1997), pp. 159-178.

[37] Y. Lin, V. Thomée and L.B. Wahlbin, Ritz-Volterra projections to finite-element spaces and applications to integrodifferential and related equations, SIAM J. Numer. Anal., 28 (1991), pp. 1047-1070.

[38] A. Lunardi, Analytic Semigroups and Optimal Regularity in Parabolic Problems, Birkhäuser Verlag, Basel, 1995.

[39] J. A. Nitsche and M. F. Wheeler, $L_{\infty}$-boundedness of the finite element Galerkin operator for parabolic problems, Numer. Funct. Anal. Optimization, 4 (1981/82), pp. 325-353.

[40] C. Palencia, Maximum norm analysis of completely discrete finite element methods for parabolic problems, SIAM J. Numer. Anal., 33 (1996), 1654-1668.

[41] R. Rannacher and R. Scott, Some optimal error estimates for piecewise linear finite element approximations, Math. Comput., 38 (1982), pp. 437-445.

[42] A. H. Schatz, V. Thomée and L. B. Wahlbin, Maximum norm stability and error estimates in parabolic finite element equations, Comm. Pure Appl. Math., 33 (1980), pp. 265-304.

[43] A. H. Schatz, V. Thomée and L. B. Wahlbin, Stability, analyticity, and almost best approximation in maximum norm for parabolic finite element equations, Comm. Pure Appl. Math., 51 (1998), pp. 1349-1385.

[44] A. E. Scheidegger, The physics of flow through porous media, The MacMillan Company, New York, 1957.

[45] S. Sun and M. F. Wheeler, Discontinuous Galerkin methods for coupled flow and reactive transport problems, Appl. Numer. Math., 52 (2005), pp. 273-298.

[46] L. Wahlbin: Local behavior in finite element methods, pp. 353-522 in: Handbook of Numerical Analysis II: Finite Element Methods (Part 1), P. G. Ciarlet and J. L. Lions, eds., North-Holland, Amsterdam, 1991.

[47] H. Wang, An optimal-order error estimate for a family of ELLAM-MFEM approximations to porous medium flow, SIAM J. Numer. Anal., 46 (2008), pp. 2133-2152.

[48] H. Wang, D. Liang, R. E. Ewing, S. L. Lyons, and G. Qin, An approximation to miscible fluid flows in porous media with point sources and sinks by an Eulerian-Lagrangian localized adjoint method and mixed finite element methods, SIAM J. Sci. Comput., 22 (2000), pp. 561-581.

[49] J. Wang, Z. Si and W. Sun, A new error analysis on characteristic methods for incompressible miscible flow in porous media, SIAM J. Numer. Anal., 52(2014), pp. 3300-3020.

[50] M. F. Wheeler, A priori $L^{2}$ error estimates for Galerkin approximations to parabolic partial differential equations, SIAM J. Numer. Anal., 10 (1973), pp. 723-759. 


\section{Appendix: Proof of Lemmas 3.23 .7}

Proof of Lemma 3.2. (3.11) and (3.12) can be found in [32, (1.18)] and [32, (2.4)], respectively. We prove (3.13) by using [32, (2.3)], which implies (via using inverse inequality)

$$
\begin{aligned}
& \left\|\mathbf{P}_{h} \Phi^{n}-\phi_{h}^{n}\right\|_{L^{p}\left(W^{1, q}\right)} \\
& \leq C h^{-1}\left\|\mathbf{P}_{h} \Phi^{n}-\phi_{h}^{n}\right\|_{L^{p}\left(L^{q}\right)} \\
& \leq C h^{-1}\left(\left\|\mathbf{P}_{h} \Phi^{n}-\mathbf{R}_{h} \Phi^{n}\right\|_{L^{p}\left(L^{q}\right)}+\left\|\mathbf{P}_{h} \Phi^{0}-\phi_{h}^{0}\right\|_{L^{q}}\right) \quad \text { (use [32, (2.3)]) } \\
& \leq C h^{-1}\left\|\Phi^{n}-\mathbf{R}_{h} \Phi^{n}\right\|_{L^{p}\left(L^{q}\right)}+C h^{-1}\left\|\mathbf{P}_{h} \Phi^{0}-\phi_{h}^{0}\right\|_{L^{q}} \quad \text { (use } L^{q} \text { stability of } \mathbf{P}_{h} \text { ) } \\
& \leq C\left\|\Phi^{n}-\mathbf{R}_{h} \Phi^{n}\right\|_{L^{p}\left(W^{1, q}\right)}+C h^{-1}\left\|\mathbf{P}_{h} \Phi^{0}-\phi_{h}^{0}\right\|_{L^{q}} . \quad \text { (use (3.2) with } l=0 \text { ) }
\end{aligned}
$$

From (3.5) and (3.6) we derive

$$
\begin{aligned}
& \left(D_{\tau}\left(\mathbf{P}_{h} \Phi^{n}-\phi_{h}^{n}\right), v_{h}\right)+\left(a(\cdot, t) \nabla\left(\mathbf{P}_{h} \Phi^{n}-\phi_{h}^{n}\right), \nabla v_{h}\right)+\left(\mathbf{P}_{h} \Phi^{n}-\phi_{h}^{n}, v_{h}\right) \\
= & \left(a(\cdot, t) \nabla\left(\mathbf{P}_{h} \Phi^{n}-\mathbf{R}_{h} \Phi^{n}\right), \nabla v_{h}\right), \quad n=1, \ldots, N,
\end{aligned}
$$

which implies

$$
\begin{aligned}
\left\|D_{\tau}\left(\mathbf{P}_{h} \Phi^{n}-\phi_{h}^{n}\right)\right\|_{L^{p}\left(\widetilde{W}^{-1, q}\right) \leq} & C\left\|a(\cdot, t) \nabla\left(\mathbf{P}_{h} \Phi^{n}-\phi_{h}^{n}\right)\right\|_{L^{p}\left(L^{q}\right)}+C\left\|\mathbf{P}_{h} \Phi^{n}-\phi_{h}^{n}\right\|_{L^{p}\left(L^{q}\right)} \\
& +C\left\|a(\cdot, t) \nabla\left(\mathbf{P}_{h} \Phi^{n}-\mathbf{R}_{h} \Phi^{n}\right)\right\|_{L^{p}\left(L^{q}\right)} \\
\leq & C\left\|\mathbf{P}_{h} \Phi^{n}-\phi_{h}^{n}\right\|_{L^{p}\left(W^{1, q}\right)}+C\left\|\mathbf{P}_{h}\left(\Phi^{n}-\mathbf{R}_{h} \Phi^{n}\right)\right\|_{L^{p}\left(W^{1, q}\right)} \\
\leq & C\left\|\Phi^{n}-\mathbf{R}_{h} \Phi^{n}\right\|_{L^{p}\left(W^{1, q}\right)}+C h^{-1}\left\|\mathbf{P}_{h} \Phi^{0}-\phi_{h}^{0}\right\|_{L^{q}},
\end{aligned}
$$

where we have used (A.1) in the last inequality. The proof is completed.

\section{Proof of Lemma 3.3 .}

(1) Under the conditions of Lemma 3.3, the Lax-Milgram lemma implies that (3.14) has a unique weak solution $u \in H^{1} \hookrightarrow L^{6}$ under the constraint $\int_{\Omega} u \mathrm{~d} x=0$, satisfying $\|u\|_{H^{1}} \leq C\|f\|_{L^{2}}$. Thus $u$ is also a weak solution of

$$
\begin{cases}\sum_{i, j=1}^{d} \frac{\partial}{\partial x_{i}}\left(a_{i j} \frac{\partial u}{\partial x_{j}}\right)-u=f-u & \text { in } \Omega \\ \sum_{i, j=1}^{d} a_{i j} n_{i} \partial_{j} u=0 & \text { on } \partial \Omega,\end{cases}
$$

which satisfies the following estimate (applying [18, Theorem 2.4.2.7] with $p=2$ )

$$
\begin{aligned}
\|u\|_{H^{2}} \leq C\|f-u\|_{L^{2}} & \leq C\left(\|f\|_{L^{2}}+\|u\|_{L^{2}}\right) \\
& \leq C\left(\|f\|_{L^{2}}+\|u\|_{H^{1}}\right) \\
& \leq C\|f\|_{L^{2}} .
\end{aligned}
$$

Since $H^{2} \hookrightarrow L^{\infty}$ in both two- and three-dimensional spaces, we have

$$
\|u\|_{L^{\infty}} \leq\|u\|_{H^{2}} \leq C\|f\|_{L^{2}}
$$

Applying [18, Theorem 2.4.2.7] again yields

$$
\begin{aligned}
\|u\|_{W^{2, q}} & \leq C_{q}\|f+u\|_{L^{q}} \\
& \leq C_{q}\left(\|f\|_{L^{q}}+\|u\|_{L^{q}}\right) \\
& \leq C_{q}\left(\|f\|_{L^{q}}+\|u\|_{L^{\infty}}\right) \\
& \leq C_{q}\left(\|f\|_{L^{q}}+\|f\|_{L^{2}}\right) \\
& \leq C_{q}\|f\|_{L^{q}} .
\end{aligned}
$$

This proves (3.15). 
(2) By choosing $q>d$ we have $f \in C^{\alpha} \hookrightarrow L^{q}$. (3.15) implies $u \in W^{2, q} \hookrightarrow C^{1, \alpha} \hookrightarrow C^{\alpha}$ with $\alpha=1-d / q \in(0,1)$. Thus $u$ is also a solution of

$$
\begin{cases}\sum_{i, j=1}^{d} \frac{\partial}{\partial x_{i}}\left(a_{i j} \frac{\partial u}{\partial x_{j}}\right)-u=f-u \in C^{\alpha} & \text { in } \Omega, \\ \sum_{i, j=1}^{d} a_{i j} n_{i} \partial_{j} u=0 & \text { on } \partial \Omega,\end{cases}
$$

which satisfies the following Hölder estimate (applying [35, Theorem 4.40 and Corollary 4.41])

$$
\|u\|_{C^{2, \alpha}} \leq C\|f-u\|_{C^{\alpha}} \leq C\left(\|f\|_{C^{\alpha}}+\|u\|_{C^{\alpha}}\right) \leq C\|f\|_{C^{\alpha}} .
$$

This completes the proof of Lemma 3.16 .

Proof of Lemma 3.4. Since $f \in C^{\alpha} \hookrightarrow L^{2}$, the Lax-Milgram lemma implies the existence of a unique weak solution $u \in \stackrel{\circ}{H}^{1} \hookrightarrow L^{6}$, and the $W^{1, s}$ estimate of elliptic equations (cf. [5. Theorem 1]) implies $\|u\|_{W^{1, d+1}} \leq C\|f\|_{L^{d+1}}$. Since $W^{1, d+1} \hookrightarrow L^{\infty}$, it follows that

$$
\|u\|_{L^{\infty}} \leq C\left(\|g\|_{L^{\infty}}+\|f\|_{L^{d+1}}\right) \leq C\left(\|g\|_{L^{\infty}}+\|f\|_{C^{\alpha}}\right) \text {. }
$$

Let $\chi=\chi(t)$ be a smooth cut-off function defined for $t \in[0,2]$ such that $\chi(t)=1$ for $t \in[1,2]$ and $\chi(0)=0$, satisfying $\left|\partial_{t} \chi\right| \leq C$. Then $\chi u$ satisfies the parabolic equation ( $u$ is time-independent)

$$
\begin{cases}\partial_{t}(\chi u)-\sum_{i, j=1}^{d} \frac{\partial}{\partial x_{i}}\left(a_{i j} \frac{\partial(\chi u)}{\partial x_{j}}\right)=u \partial_{t} \chi+\chi g+\sum_{i=1}^{d} \partial_{i}\left(\chi f_{i}\right) & \text { in } \Omega \times[0,2], \\ \sum_{i, j=1}^{d} a_{i j} n_{i} \frac{\partial(\chi u)}{\partial x_{j}}=\sum_{i=1}^{d} \chi f_{i} n_{i} & \text { on } \partial \Omega \times[0,2], \\ \chi(0) u(x, 0)=0 & \text { for } x \in \Omega .\end{cases}
$$

[34, Theorem 4.30] immediately implies

$$
\begin{aligned}
& \|\chi u\|_{L^{\infty}\left(0,2 ; C^{1, \alpha}\right)} \\
& \leq C\left\|u \partial_{t} \chi\right\|_{L^{\infty}\left(0,2 ; L^{\infty}\right)}+C\|\chi g\|_{L^{\infty}\left(0,2 ; L^{\infty}\right)}+C\left(\|\chi f\|_{L^{\infty}\left(0,2 ; C^{\alpha}\right)}+\|\chi f\|_{C^{\alpha}\left(0,2 ; L^{\infty}\right)}\right) \\
& \leq C\|u\|_{L^{\infty}}+C\|g\|_{L^{\infty}}+C\|f\|_{C^{\alpha}} \\
& \leq C\left(\|g\|_{L^{\infty}}+\|f\|_{C^{\alpha}}\right)
\end{aligned}
$$

where the last inequality is due to (A.10). Since $\chi$ is independent of the $x$ variable and $u$ is independent of the $t$ variable, it follows that

Thus (A.12) implies

$$
\|\chi u\|_{L^{\infty}\left(0,2 ; C^{1, \alpha}\right)}=\|\chi\|_{L^{\infty}}\|u\|_{C^{1, \alpha}} .
$$

This completes the proof of Lemma 3.4

$$
\|u\|_{C^{1, \alpha}} \leq C\left(\|g\|_{L^{\infty}}+\|f\|_{C^{\alpha}}\right) .
$$

Proof of Lemma 3.5 The existence and uniqueness of solution $u_{h} \in \stackrel{\circ}{S}_{h}^{r}$ is standard. It suffices to prove the estimate (3.19). Note that (3.18) is equivalent to

$$
\left(a \nabla u_{h}, \nabla v_{h}\right)+\left(u_{h}, v_{h}\right)=\left(\mathbf{f}, \nabla v_{h}\right)+\left(u_{h}, v_{h}\right), \quad \forall v_{h} \in \stackrel{\circ}{S}_{h}^{r} .
$$

Let $u \in H^{1}$ be the solution of the PDE problem

$$
\begin{cases}-\nabla \cdot(a \nabla u)+u=-\nabla \cdot \mathbf{f}+u_{h} & \text { in } \Omega, \\ a \nabla u \cdot \mathbf{n}=\mathbf{f} \cdot \mathbf{n} & \text { on } \partial \Omega,\end{cases}
$$

so that $u_{h}$ is the Ritz projection of $u$. Then the $W^{1, q}$ stability of Ritz projections (as an interpolation [17, Corollary A.6]) says that

$$
\left\|u_{h}\right\|_{W^{1, q}} \leq C\|u\|_{W^{1, q}}
$$


and the $W^{1, q}$ estimate of elliptic equations (cf. [5. Theorem 1]) says that

$$
\|u\|_{W^{1, q}} \leq C_{q}\left(\|\mathbf{f}\|_{L^{q}}+\left\|u_{h}\right\|_{L^{q}}\right) \leq C_{q}\|\mathbf{f}\|_{L^{q}}+C_{q, \epsilon}\left\|u_{h}\right\|_{L^{2}}+\epsilon\left\|u_{h}\right\|_{W^{1, q}},
$$

where $\epsilon \in(0,1)$ can be arbitrarily small at the expense of enlarging the constant $C_{q, \epsilon}$. The two estimates above imply

$$
\begin{aligned}
\left\|u_{h}\right\|_{W^{1, q}} & \leq C_{q}\|\mathbf{f}\|_{L^{q}}+C_{q}\left\|u_{h}\right\|_{L^{2}} \\
& \leq C_{q}\|\mathbf{f}\|_{L^{q}}+C_{q}\left\|u_{h}\right\|_{H^{1}} \\
& \leq C_{q}\|\mathbf{f}\|_{L^{q}}+C_{q}\|\mathbf{f}\|_{L^{2}} \\
& \leq C_{q}\|\mathbf{f}\|_{L^{q}}
\end{aligned}
$$

Proof of Lemma 3.6 If we define

$$
\phi(t)= \begin{cases}\frac{t_{k}-t}{\tau} \phi^{k-1}+\frac{t-t_{k-1}}{\tau} \phi^{k}, & \text { for } t \in\left[t_{k-1}, t_{k}\right], \quad k=1, \ldots, n, \\ \phi\left(2 t_{n}-t\right), & \text { for } t \in\left[t_{n}, 2 t_{n}\right], \\ 0, & \text { for } t \in\left[2 t_{n}, \infty\right),\end{cases}
$$

then the function $\phi$ is piecewise linear in time and supported in the time interval $\left[0,2 t_{n}\right]$, satisfying the following estimate:

$$
\left\|\partial_{t} \phi\right\|_{L^{p}\left(\mathbb{R}_{+} ; \widetilde{W}^{-1, q}\right)}+\|\phi\|_{L^{p}\left(\mathbb{R}_{+} ; W^{1, q}\right)} \leq C\left(\left\|D_{\tau} \phi^{n}\right\|_{L^{p}\left(\widetilde{W}^{-1, q}\right)}+\left\|\phi^{n}\right\|_{L^{p}\left(W^{1, q}\right)}\right) .
$$

Let $E$ denote a global extension operator which maps $W^{1, q}$ boundedly into $W^{1, q}\left(\mathbb{R}^{d}\right)$ and maps $\widetilde{W}^{-1, q}$ boundedly into $W^{-1, q}\left(\mathbb{R}^{d}\right)$, such that $E u=u$ in $\Omega$ for all $u \in \widetilde{W}^{-1, q}$. Such an extension operator exists, by reflecting the function with respect to the boundary $\partial \Omega$; see [1, Theorems 5.19 and 5.22]. By the real interpolation method, we have

$$
\begin{aligned}
& E \text { maps }\left(\widetilde{W}^{-1, q}, W^{1, q}\right)_{1-1 / p, p} \text { boundedly into }\left(W^{-1, q}\left(\mathbb{R}^{d}\right), W^{1, q}\left(\mathbb{R}^{d}\right)\right)_{1-1 / p, p}, \\
& \left(W^{-1, q}\left(\mathbb{R}^{d}\right), W^{1, q}\left(\mathbb{R}^{d}\right)\right)_{1-1 / p, p}=B^{1-2 / p, q ; p}\left(\mathbb{R}^{d}\right) \hookrightarrow C^{\alpha}\left(\mathbb{R}^{d}\right), \text { for } \alpha \in(0,1-2 / p-d / q),
\end{aligned}
$$

where $B^{1-2 / p, q ; p}\left(\mathbb{R}^{d}\right)$ denotes the Besov space in $\mathbb{R}^{d}$ (cf. [1, $\left.\S 7.32\right]$ ), with the embedding property $B^{1-2 / p, q ; p}\left(\mathbb{R}^{d}\right) \hookrightarrow C^{\alpha}\left(\mathbb{R}^{d}\right)$ for $0<\alpha<1-2 / p-d / q$ (cf. [1, §7.34]). Then the inhomogeneous Sobolev embedding (see [38, Proposition 1.2.10])

$$
\|\phi\|_{\left.\left.L^{\infty}\left(\mathbb{R}_{+} ;\left(\widetilde{W}^{-1, q}\right), W^{1, q}\right)\right)_{1-1 / p, p}\right)} \leq C\left(\left\|\partial_{t} \phi\right\|_{L^{p}\left(\mathbb{R}_{+} ; \widetilde{W}^{-1, q}\right)}+\|\phi\|_{L^{p}\left(\mathbb{R}_{+} ; W^{1, q}\right)}\right),
$$

together with (A.20)- A.21), implies

$$
\|\phi\|_{L^{\infty}\left(\mathbb{R}_{+} ; C^{\alpha}\right)} \leq C\left(\left\|D_{\tau} \phi^{n}\right\|_{L^{p}\left(\widetilde{W}^{-1, q}\right)}+\left\|\phi^{n}\right\|_{L^{p}\left(W^{1, q}\right)}\right) .
$$

This proves (3.20).

The inequality (3.21) can be proved similarly in view of the interpolation result

$$
\left(L^{q}\left(\mathbb{R}^{d}\right), W^{2, q}\left(\mathbb{R}^{d}\right)\right)_{1-1 / p, p}=B^{2-2 / p, q ; p}\left(\mathbb{R}^{d}\right) \hookrightarrow C^{1, \alpha}\left(\mathbb{R}^{d}\right) \text {, for } \alpha \in(0,1-2 / p-d / q) .
$$

The proof of Lemma 3.6 is complete.

Proof of Lemma 3.7 Hölder's inequality implies that

$$
\begin{aligned}
\left(\tau \sum_{n=k+1}^{m}\left|Y^{n}\right|^{p}\right)^{\frac{1}{p}} & \leq \alpha\left(Y^{k}+\tau \sum_{n=k+1}^{m}\left|Y^{n}\right|\right)+\beta \\
& \leq \alpha\left(Y^{k}+\left(t_{m}-t_{k}\right)^{1-\frac{1}{p}}\left(\tau \sum_{n=k+1}^{m}\left|Y^{n}\right|^{p}\right)^{\frac{1}{p}}\right)+\beta
\end{aligned}
$$

If $\left(t_{m}-t_{k}\right)^{1-\frac{1}{p}} \leq(2 \alpha)^{-1}$ then the last inequality is reduced to

$$
\left(\tau \sum_{n=k+1}^{m}\left|Y^{n}\right|^{p}\right)^{\frac{1}{p}} \leq 2 \alpha Y^{k-1}+2 \beta .
$$


Let $\tau_{p}=\frac{1}{4(2 \alpha)^{1 /(1-1 / p)}}$ and $m=\left[\frac{1}{2 \tau(2 \alpha)^{1 /(1-1 / p)}}\right]$ so that $(2 m \tau)^{1-\frac{1}{p}} \leq(2 \alpha)^{-1}$, and

$$
2 m \tau=2 \tau\left[\frac{1}{2 \tau(2 \alpha)^{1 /(1-1 / p)}}\right] \geq \frac{1}{(2 \alpha)^{1 /(1-1 / p)}}-2 \tau \geq \frac{1}{2(2 \alpha)^{1 /(1-1 / p)}}, \quad \text { for } \tau \leq \tau_{p} .
$$

We choose a sequence $0=t_{n_{0}}<t_{n_{1}}<\cdots<t_{n_{\ell}}=T$ (so $n_{\ell}=N$ ) in the following way.

If $t_{n_{j}}+2 m \tau \geq T$ then we choose $t_{n_{j+1}}=T$.

If $t_{n_{j}}+2 m \tau<T$ then we choose $t_{n_{j+1}} \in\left[t_{n_{j}}+m \tau, t_{n_{j}}+2 m \tau\right]$ such that

$$
Y^{n_{j+1}}=\min _{n_{j}+m+1 \leq n \leq n_{j}+2 m} Y^{n}
$$

Then

$$
Y^{n_{j+1}} \leq\left(\frac{1}{m} \sum_{n=n_{j}+m+1}^{n_{j}+2 m}\left|Y^{n}\right|^{p}\right)^{\frac{1}{p}}=\left(\frac{1}{m \tau} \tau \sum_{n=n_{j}+m}^{n_{j}+2 m}\left|Y^{n}\right|^{p}\right)^{\frac{1}{p}} \leq(m \tau)^{-\frac{1}{p}}\left(\tau \sum_{n=n_{j}+1}^{n_{j}+2 m}\left|Y^{n}\right|^{p}\right)^{\frac{1}{p}},
$$

and (A.25) implies

$$
\left(\tau \sum_{n=n_{j}+1}^{n_{j}+2 m}\left|Y^{n}\right|^{p}\right)^{\frac{1}{p}} \leq 2 \alpha Y^{n_{j}}+2 \beta
$$

The last two estimates show that

$$
\begin{aligned}
Y^{n_{j+1}} & \leq 2^{\frac{1}{p}} \Delta T^{-\frac{1}{p}}\left(\tau \sum_{n=n_{j}+1}^{n_{j}+2 m}\left|Y^{n}\right|^{p}\right)^{\frac{1}{p}} \\
& \leq 2^{1+\frac{1}{p}} \Delta T^{-\frac{1}{p}} \alpha Y^{n_{j}}+2^{1+\frac{1}{p}} \Delta T^{-\frac{1}{p}} \beta \\
& \leq C_{\alpha, p} Y^{n_{j}}+C_{\alpha, p} \beta .
\end{aligned}
$$

Iterations of the above two estimates give (the number of iterations is bounded by $2(2 \alpha)^{1 /(1-1 / p)} T$ )

$$
\begin{aligned}
& \max _{0 \leq j \leq \ell-1} Y^{n_{j}} \leq C_{T, \alpha, p}\left(Y^{0}+\beta\right), \\
& \max _{0 \leq j \leq \ell-1}\left(\tau \sum_{n=n_{j}+1}^{n_{j}+2 m}\left|Y^{n}\right|^{p}\right)^{\frac{1}{p}} \leq C_{T, \alpha, p}\left(Y^{0}+\beta\right),
\end{aligned}
$$

and applying (A.26) again yields

$$
\left(\tau \sum_{n=n_{\ell-1}+1}^{n_{\ell}}\left|Y^{n}\right|^{p}\right)^{\frac{1}{p}} \leq 2 \alpha Y^{n_{\ell-1}}+2 \beta \leq C_{T, \alpha, p}\left(Y^{0}+\beta\right) .
$$

Since $\ell \leq 1+2(2 \alpha)^{1 /(1-1 / p)} T$ (a bounded number independent of $\tau$ ), the last two inequalities imply (3.23). This completes the proof of Lemma 3.7

Department of Mathematics, School of Sciences, Hangzhou Dianzi University, Hangzhou 310018, CHINA

E-mail address: femwentao@hdu.edu.cn

Department of Applied Mathematics, The Hong Kong Polytechnic University, Kowloon, Hong Kong E-mail address: bygli@polyu.edu.hk

Department of Applied Mathematics, The Hong Kong Polytechnic University, Kowloon, Hong KONG. E-MAIL: YANPING.LIN@ POLYU.EDU.HK

E-mail address: yanping.lin@polyu.edu.hk

Department of Mathematics, City University of Hong Kong, Kowloon, Hong Kong.

E-mail address: maweiw@cityu.edu.hk 